\title{
Speciation of 38 elements in eight GSJ geochemical sedimentary reference materials determined using a sequential extraction technique
}

\author{
RAN Kubota*, AtsuYUKi OHTA and TAKASHI OKAI \\ Geological Survey of Japan, AIST, Ibaraki 305-8567, Japan
}

(Received January 24, 2013; Accepted November 22, 2013)

\begin{abstract}
A sequential extraction procedure developed by the European Community Bureau of Reference (BCR) was applied to eight sediment geochemical reference materials provided by the Geological Survey of Japan, AIST (JLk-1, JSd-1-4, JMs1, JMs-2, and JSO-1). The study aimed to provide reference materials of widely varied geological materials in order to enhance the utility of the sequential extraction procedure in environmental studies. The concentrations of 38 elements extracted by BCR protocols were determined for the eight reference materials. The BCR scheme is designed to extract elements of the intended phase using acetic acid (step 1), hydroxylammonium chloride (step 2), and hydrogen peroxide and ammonium acetate (step 3). The residue of the step 3 extraction was further digested using hydrofluoric acid, perchloric acid, and nitric acid (step 4). The relative standard deviations of elemental concentrations in each extraction stage were generally $<30 \%$ for steps 1 and 3 and $<20 \%$ for steps 2 and 4 . The sums of the elemental concentrations for the four steps (the total recoveries) ranged from $70 \%$ to $100 \%$ of the bulk compositions. The speciation of elements in the reference materials obtained by BCR protocols was strongly affected by sediment origin, mineralogical composition, sedimentary environment, and anthropogenic activity. Accordingly, sequential extraction based on the BCR procedure is effective for environmental studies. Eight geochemical reference materials certified/recommended for bulk composition are now further available as reference materials for the sequential extraction procedure.
\end{abstract}

Keywords: BCR protocol, geochemical reference material, sequential extraction, speciation

\section{INTRODUCTION}

The distribution of elemental abundance in the earth's surface is fundamental and essential information for mineral exploration and environmental assessment because the surface layer relates closely to the living beings that exist there. Spatial distribution patterns of elemental concentrations (geochemical maps), even at a subcontinental scale, have been prepared in many countries for various purposes (e.g., Fauth et al., 1985; Lahermo et al., 1990; Lis and Pasieczna, 1995; Reimann et al., 1998; Salminen et al., 2005; Weaver et al., 1983; Webb et al., 1978; Xie et al., 1997). In Japan, the Geological Survey of Japan, National Institute of Advanced Industrial Science and Technology (AIST), conducted nationwide geochemical mapping at 1:2,000,000 scale using stream and marine sediments (Imai et al., 2004, 2010). The purpose of the map is to obtain natural geochemical baselines on the surface of the earth. In order to evaluate the effects of anthropogenic pollution, it is necessary to accurately understand the background values of naturally

*Corresponding author (e-mail: ran-kubota@aist.go.jp) Copyright @ 2014 by The Geochemical Society of Japan. occurring elements. For this purpose, in terrestrial areas, stream sediment has been used for making geochemical maps. Stream sediment has been used because its geochemistry is assumed to represent the averaged composite elemental composition of the watershed (e.g., Howarth and Thornton, 1983). On the other hand, rock has not been used as sample media because its geochemistry reflects only the local area, is too inhomogeneous to create nationwide geochemical maps, and provides no information about contamination.

Ohta et al. $(2005,2011)$ successfully identified contamination by $\mathrm{P}, \mathrm{Cu}, \mathrm{Zn}, \mathrm{As}, \mathrm{Mo}, \mathrm{Cd}, \mathrm{Sn}, \mathrm{Sb}, \mathrm{Hg}, \mathrm{Pb}$, and $\mathrm{Bi}$ in stream sediments collected from urban areas by applying a statistical test to the geochemical data. However, some parent lithologies elevate the concentrations of these elements in stream sediments. For example, stream sediments originated from ultramafic rock contain several thousand milligrams per kilogram of $\mathrm{Cr}$ and $\mathrm{Ni}$ (Ohta et al., 2005). In addition, an element in sediments can occur in various physicochemical forms: exchangeable ion, adsorbed ion, carbonate, $\mathrm{Fe}-\mathrm{Mn}$ oxide, sulfide, organic matter, mineral lattice, and so on (e.g., Tessier $e t$ al., 1979). The toxicity of pollutants also changes with their chemical species. For these reasons, the bulk composition provided by geochemical maps is insufficient to elucidate a risk assessment for heavy metals. In areas 
Table 1. List of 8 Geological Survey of Japanese reference materials

\begin{tabular}{|c|c|c|c|c|}
\hline Name & Category & Preparation & Note & Reference \\
\hline JLk-1 & Lake sediment & 1987 & Muddy lake sediments collected from the Lake Biwa & Ando et al. (1990) \\
\hline JSd-1 & Stream sediment & 1988 & Originating from granitic rocks & Terashima et al. (1990) \\
\hline JSd-2 & Stream sediment & 1989 & Originating from metamorphic rocks associated with Hitachi $\mathrm{Cu}$ mine & Terashima et al. (1990) \\
\hline JSd-3 & Stream sediment & 1989 & Originating from sedimentary rocks (melange matrix of accretionary complexes) & Terashima et al. (1990) \\
\hline JSd-4 & Stream sediment & 2010 & Collected from alluvial plain in Kanto District & \\
\hline JMs-1 & Marine sediment & 1999 & Muddy clay-rich marine sediments collected from the Tokyo Bay & Terashima et al. (2002) \\
\hline JMs-2 & Marine sediment & 2000 & Pelagic and zeolitic clay collected from Penrhyn Basin in the South Pacific & Terashima et al. (2002) \\
\hline JSO-1 & Soil & 1997 & Kuroboku soils (Andosol) originated from volcanic ash and rich in organic materials & Terashima et al. (2002) \\
\hline
\end{tabular}

where speciation geochemical maps have been prepared, it is possible to explore mineral occurrence and elucidate the potential hazard of toxic elements more directly (Ohta et al., 2007).

Imai et al. (2004) prepared simple speciation geochemical maps using the $0.1 \mathrm{M}$ hydrochloric acid soluble fraction of elements in stream sediments. Although this method has the advantage of being a simple and rapid procedure, it is unclear which components are extracted. Alternatively, sequential extraction schemes, such as a five-stage Tessier protocol (Tessier et al., 1979), have been widely used as effective speciation techniques. The disadvantage of sequential extraction methods is that the results obtained from different schemes are not always consistent with one another, even in the same laboratories (López-Sánchez et al., 1993; Mester et al., 1998; Usero et al., 1998). Thus, standardization of the sequential extraction procedure and interlaboratory comparison has been evaluated for the three-step extraction procedure of lake sediments developed by the Community Bureau of Reference (BCR) (Crosland et al., 1993; Ure et al., 1993). The precision and accuracy of the analytical data for elemental concentrations extracted using the BCR scheme are confirmed using reference material BCR701. BCR-701 was prepared using lake sediment (Lake Orta Piemonte, Italy) and was certified for $\mathrm{Cd}, \mathrm{Cr}, \mathrm{Cu}$, $\mathrm{Ni}, \mathrm{Pb}$, and $\mathrm{Zn}$ concentrations for the three steps (Sutherland, 2010). Unfortunately, it is the only currently available reference material for the sequential extraction procedure. The availability of a wide variety of reference materials is important for quality control of speciation studies. Indeed, future speciation geochemical mapping of the earth's surface requires a wide range of reference materials, such as stream sediment, marine sediment, and soil, for speciation. Thus, we applied the BCR protocol to sediment geochemical reference materials provided by the Geological Survey of Japan, AIST. The provision of reference values for many elements extracted by sequential extraction would be beneficial for many geochemists who conduct speciation geochemistry of sediments.

\section{SAMPLE MATERIALS}

Eight Japanese sediment geochemical reference materials were used for this sequential extraction study. Table 1 summarizes their features, origin, and sedimentary environment. JLk-1 is a lake sediment collected from Lake Biwa, which is the largest lake in Japan (Ando et al., 1990). JSd-1-4 are stream sediment reference materials collected from drainage basins with granitic rocks (JSd1), the Hitachi Copper Mine (JSd-2), sedimentary rocks (JSd-3), and an alluvial plain in the Kanto District (JSd4) (Terashima et al., 1990). JMs-1 and JMs-2 are marine sediments collected from Tokyo Bay and the Penrhyn Basin (South Pacific Ocean), respectively (Terashima $e t$ al., 2002). JSO-1 is a soil reference material, categorized as an andosol (Kuroboku) soil (Terashima et al., 2002). All materials were well dried in the ambient condition; however, JSO- 1 and JMs-1 and JMs-2 were further dried for one week at about $40^{\circ} \mathrm{C}$ (Terashima et al., 2002) at the time of issue. These reference samples were not intended for speciation chemistry of elements, and the possibility that changes of speciation of elements in soil materials could occur during drying has been suggested. However, the long-term stability of extracted results has been confirmed in dried certified reference materials (Rauret et al., 2000). Therefore, the speciation of elements in our reference materials archived in dried conditions also should not change after their release. We also addressed the binding fraction of the elements in each sample from the stepwise leaching technique. The precision and accuracy of the analytical results for elemental concentrations extracted by the BCR scheme were confirmed using certified reference material BCR-701, which was obtained from the Institute for Reference Materials and Measurements (IRMM).

\section{EXPERIMENTAL Methods}

The sequential extraction was performed in conformity with the BCR scheme proposed by Thomas et al. 


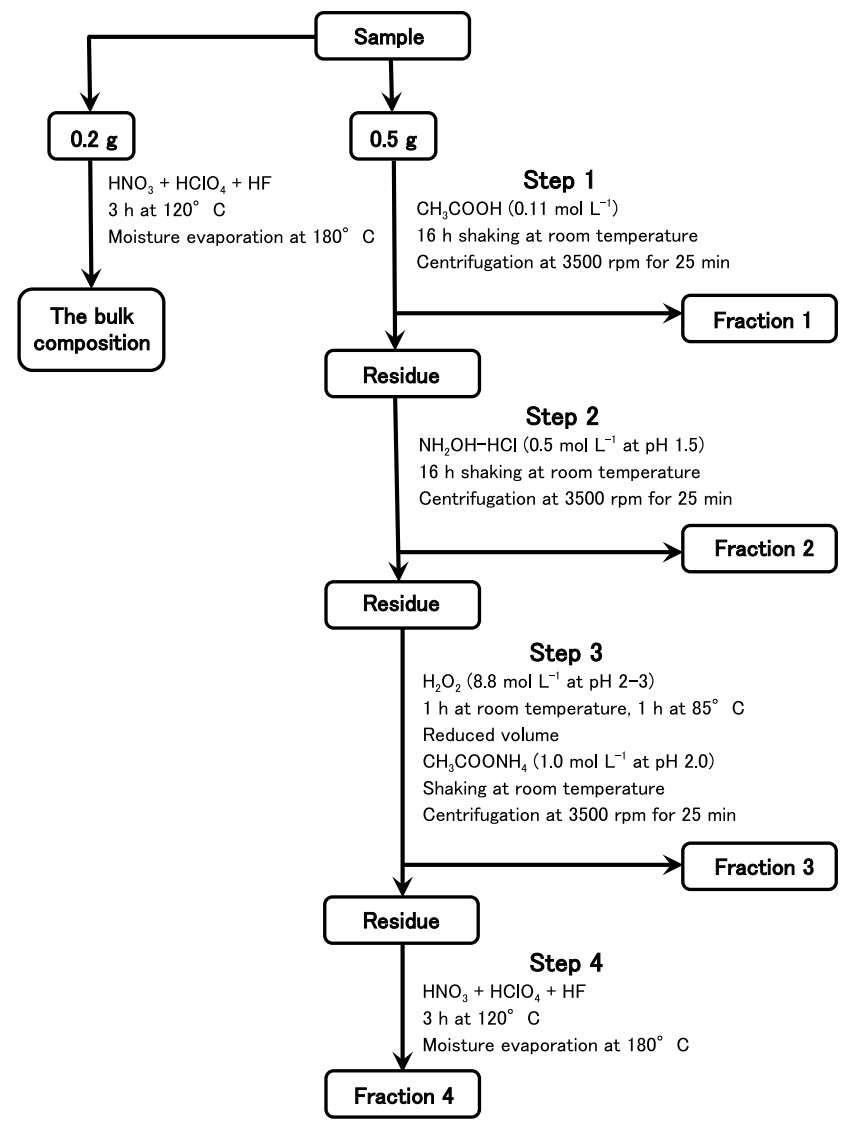

Fig. 1. Flow diagram of the BCR sequential extraction protocol.

(1994) and Ure et al. (1993) and modified by Rauret et al. (1999) and Sahuquillo et al. (1999). Thirty-eight elements were extracted from the eight sediment geochemical reference materials and BCR701 based on the BCR scheme (defined by Rauret et al. (1999)), except that a $0.5 \mathrm{~g}$ sample was used instead of a $1 \mathrm{~g}$ sample and the shaking was performed at $80 \mathrm{rpm}$ because the sample was not suspended in solution at $30 \mathrm{rpm}$. Furthermore, the water used for washing the residue was added into the extracted solution without discarding to improve the recovery rate. A brief summary of the method, shown schematically in Fig. 1, is given in the following section. The extracting reagents such as acetic acid, hydroxyammonium chloride, hydrogen peroxide, and ammonium acetate were prepared from analytical grade according to Rauret et al. (1999). Deionized ultrapure water (e.g., Milli-Q water) was used throughout.

\section{Sequential extraction procedure (the BCR scheme)}

Step 1: This fractionation intends to extract elements in carbonates or those weakly adsorbed on materials (exchangeable elements). $0.5 \mathrm{~g}$ of powdered sample, which was dried at $105^{\circ} \mathrm{C}$ for $3 \mathrm{~h}$, is added to a $50 \mathrm{~mL}$ PFA tube. $20 \mathrm{~mL}$ of acetic acid $\left(0.11 \mathrm{~mol} \mathrm{~L}^{-1}\right)$ is then added to the cool material and a screw-on lid is placed on the PFA tube. The PFA tube is shaken for $16 \mathrm{~h}$ at room temperature $\left(25 \pm 2{ }^{\circ} \mathrm{C}\right)$ in an end-over-end mechanical shaker at a speed of $80 \mathrm{rpm}$ to suspend the sample in the solution. The sample sank to the bottom of the PFA tube at a speed of $30 \mathrm{rpm}$, which was proposed in the original protocol (Rauret et al., 1999). Supernatant solution is then separated from the solid residue by centrifugation at $3500 \mathrm{rpm}$ for $25 \mathrm{~min}$, removed with a pipette, and then filtrated using a cellulose acetate-type membrane filter $(\varnothing=0.2 \mu \mathrm{m})$. The solution is stored in a $100 \mathrm{~mL}$ polyethylene volumetric flask. The residue is washed with $10 \mathrm{~mL}$ of water by shaking for $15 \mathrm{~min}(80 \mathrm{rpm})$ and then centrifuged. The wash solution is filtered into the volumetric flask containing the first extracted solution. Finally, $5 \mathrm{~mL}$ of $7 \mathrm{~mol}$ $\mathrm{L}^{-1}$ nitric acid is added to the solution and it is made up to $100 \mathrm{~mL}$ with water.

Step 2: This fractionation is used to extract the reducible phase and target the elements bound to iron and manganese oxides that would be released when the redox condition changes. Hydroxylammonium chloride solution $\left(0.5 \mathrm{~mol} \mathrm{~L}^{-1}\right.$, adjusted to $\mathrm{pH} 1.5$ with $1 \mathrm{~mol} \mathrm{~L}^{-1}$ nitric acid) is prepared immediately before use, and $20 \mathrm{~mL}$ of this reagent is added to the residue obtained from step 1 . The subsequent extraction procedure is performed as in the case of step $1.12 .5 \mathrm{~mL}$ of $7 \mathrm{~mol} \mathrm{~L}^{-1}$ nitric acid is then added to the supernatant solution and it is made up to $250 \mathrm{~mL}$ with water in a polyethylene volumetric flask.

Step 3: This fractionation pertains to the oxidizable phase. Metal sulfides and metals bound to organic matter that would be released into the environment under oxidizing conditions are extracted in this process. $5 \mathrm{~mL}$ of hydrogen peroxide $\left(8.8 \mathrm{~mol} \mathrm{~L}^{-1}\right.$, adjusted to $\mathrm{pH} 2-3$ with $1 \mathrm{~mol} \mathrm{~L}^{-1}$ nitric acid) is added, in small aliquots, to the residue of step 2 in the centrifuge tube. The screw-on lid on the PFA tube is loosened to release the gases produced during reaction with sulfides and organic matter. In order to avoid violent reactions, the PFA tube is put in a water bath for moderate digestion at ambient temperature (20$30^{\circ} \mathrm{C}$ ) with occasional manual shaking for $1 \mathrm{~h}$. Subsequently, the tube is put in a modular heating block and heated on a hot plate at $85^{\circ} \mathrm{C}$ for $1 \mathrm{~h}$. The screw-on lid is then removed and the tube contents are evaporated to a small volume $(\sim 0.5 \mathrm{~mL})$. After cooling, a second aliquot of $5 \mathrm{~mL}$ of hydrogen peroxide is added and the same procedure is repeated. $25 \mathrm{~mL}$ of ammonium acetate solution (1.0 mol L $^{-1}$, adjusted to $\mathrm{pH} 2.0$ with conc. nitric acid) is then added to the cool residue and a screw-on lid is put on the PFA tube. The tube is shaken for $16 \mathrm{~h}$ at room temperature $\left(25 \pm 2^{\circ} \mathrm{C}\right)$ on the shaker, which is operated at a speed of $80 \mathrm{rpm}$. The extraction procedure and separation of supernatant are repeated as in the case of step 1 . 
$12.5 \mathrm{~mL}$ of $7 \mathrm{~mol} \mathrm{~L}^{-1}$ nitric acid is then added to the supernatant solution and it is made up to $250 \mathrm{~mL}$ with water in a polyethylene volumetric flask.

Step 4: This fractionation is associated with the crystalline structure of minerals. This fraction is not part of the target for the BCR extraction scheme, so we call the residue of the step 3 extraction "step 4." $10 \mathrm{~mL}$ of hydrofluoric acid, $6 \mathrm{~mL}$ of nitric acid, and $4 \mathrm{~mL}$ of perchloric acid are added to the residue of step 3 in the PFA tube. The tube, with its screw-on lid loosely attached, is put in a modular heating block on a hot plate at $120^{\circ} \mathrm{C}$ for $3 \mathrm{~h}$. The solution is then evaporated to dryness, with occasional stirring, at $180^{\circ} \mathrm{C}$. The dried residue is dissolved in $5 \mathrm{~mL}$ of $7 \mathrm{~mol} \mathrm{~L}^{-1}$ nitric acid on a hot plate at about $100^{\circ} \mathrm{C}$ and made up to $100 \mathrm{~mL}$ in a polyethylene volumetric flask; $5 \mathrm{~mL}$ of the solution and $2.2 \mathrm{~mL}$ of 7 mol $\mathrm{L}^{-1}$ nitric acid are then poured into a polyethylene volumetric flask, and diluted to $50 \mathrm{~mL}$ with water.

To confirm the recovery of elements extracted by the BCR protocol, bulk compositions of samples are also determined. $0.2 \mathrm{~g}$ of powdered sample, which was dried at $105^{\circ} \mathrm{C}$ for $3 \mathrm{~h}$, is digested using an $\mathrm{HF}-\mathrm{HNO}_{3}-\mathrm{HClO}_{4}$ mixture in a Teflon beaker. The digestion is the same as the procedure of step 4 . The dried residue is then dissolved with $5 \mathrm{~mL}$ of $7 \mathrm{~mol} \mathrm{~L}^{-1}$ nitric acid and made up to $100 \mathrm{~mL}$ with water in a polyethylene volumetric flask.

\section{Determination of elements}

Concentrations of 38 elements extracted at each step were determined using ICP-AES and ICP-MS by the calibration-curve method. Although $\mathrm{As}, \mathrm{Sn}, \mathrm{Sb}$, and $\mathrm{Hg}$ are interesting subjects for environmental assessment, these were not covered in this study. The reason why these elements were not covered is because $\mathrm{As}, \mathrm{Sn}, \mathrm{Sb}$, and $\mathrm{Hg}$ (also Ti, Nb, and Ta) in stream sediments and soil materials are readsorbed on particles during the extraction procedure or scarcely extracted by the three-step BCR protocol (Gómez-Ariza et al., 1999; Marin et al., 1997; Ohta et al., 2007).

Standard and blank solutions for each extraction step were prepared as calibration solutions. Stock solutions containing the 38 elements, prepared by consecutive dilution of $1000 \mathrm{mg} \mathrm{L}^{-1}$ standard solutions, were obtained from Merck Ltd. or Kanto Chemical Co. Inc. The standard solution was prepared by appropriate dilution of the stock solution before analysis. The matrix reagents used in each step were further added to the standard solution at a concentration equal to that of the sample solution for each step. The extractant solution obtained for each step of extraction without geochemical reference materials was used for the blank solution.

For the determination of $\mathrm{Na}, \mathrm{Mg}, \mathrm{Al}, \mathrm{P}, \mathrm{K}, \mathrm{Ca}, \mathrm{V}, \mathrm{Mn}$, $\mathrm{Fe}, \mathrm{Sr}$, and $\mathrm{Ba}$, a simultaneous echelle type ICP-AES (Nippon Jarrell Ash Model IRIS Advantage AP) was used.
The respective wavelengths (axial plasma viewing) chosen for determination were as follows: Na $589.592 \mathrm{~nm}$, Mg 285.213 nm, Al 237.312 nm, P 213.618 nm, K 766.49 nm, Ca 315.887 nm, V 310.230 nm, Mn 260.569 nm, Fe $259.940 \mathrm{~nm}, \mathrm{Sr} 407.771 \mathrm{~nm}$, and Ba $455.403 \mathrm{~nm}$. The standard solution was inserted as every 10th sample for quality control, correcting drift of the signal intensity. The internal standard method was not applied to ICP-AES measurement.

The Yokokawa Analytical Systems Model HP4500 ICP-MS was used for the determination of other minor elements $\left({ }^{45} \mathrm{Sc},{ }^{53} \mathrm{Cr},{ }^{59} \mathrm{Co},{ }^{60} \mathrm{Ni},{ }^{63} \mathrm{Cu},{ }^{66} \mathrm{Zn},{ }^{85} \mathrm{Rb},{ }^{89} \mathrm{Y}\right.$, ${ }^{97} \mathrm{Mo},{ }^{111} \mathrm{Cd},{ }^{133} \mathrm{Cs},{ }^{139} \mathrm{La},{ }^{140} \mathrm{Ce},{ }^{141} \mathrm{Pr},{ }^{146} \mathrm{Nd},{ }^{147} \mathrm{Sm},{ }^{151} \mathrm{Eu}$, ${ }^{157} \mathrm{Gd},{ }^{159} \mathrm{~Tb},{ }^{163} \mathrm{Dy},{ }^{165} \mathrm{Ho},{ }^{167} \mathrm{Er},{ }^{169} \mathrm{Tm},{ }^{173} \mathrm{Yb},{ }^{175} \mathrm{Lu}$, ${ }^{208} \mathrm{~Pb}$, and $\left.{ }^{209} \mathrm{Bi}\right)$. The blank, standard and sample solutions were mixed with $100 \mu \mathrm{g} / \mathrm{L}$ indium solution and diluted fivefold with water using an automatic dilution system. The solution containing indium was prepared as an internal standard for correction of the matrix effect by coexistence of major elements and drift of the signal intensity. As described above, the standard solution was inserted as every 10th sample for quality control. The ${ }^{59} \mathrm{Co},{ }^{60} \mathrm{Ni}$, and ${ }^{63} \mathrm{Cu}$ results may be interfered respectively by ${ }^{43} \mathrm{Ca}^{16} \mathrm{O},{ }^{44} \mathrm{Ca}^{16} \mathrm{O}$, and ${ }^{40} \mathrm{Ar}^{23} \mathrm{Na}$. Incidentally, the oxide generation rates of ${ }^{43} \mathrm{Ca}$ and ${ }^{44} \mathrm{Ca}$ were $0.1 \%$ and $0.05 \%$ respectively. Therefore, this interference was negligible in most cases. Exceptionally, the count of ${ }^{60} \mathrm{Ni}$ in JMs-2 extracted at step 1 increased to $6 \%$ because of the interference of ${ }^{44} \mathrm{Ca}^{16} \mathrm{O}$. The $\mathrm{Ni}$ concentration was corrected appropriately. The interference of ${ }^{151} \mathrm{Eu},{ }^{157} \mathrm{Gd}$, and ${ }^{159} \mathrm{~Tb}$ by ${ }^{135} \mathrm{Ba}^{16} \mathrm{O},{ }^{141} \mathrm{Pr}^{16} \mathrm{O}$, and ${ }^{143} \mathrm{Nd}^{16} \mathrm{O}$ were not corrected in this study. The oxide generation rates of ${ }^{135} \mathrm{Ba}$, ${ }^{141} \mathrm{Pr}$, and ${ }^{143} \mathrm{Nd}$ were $0.08 \%, 0.8 \%$, and $0.06 \%$, respectively. Because the $\mathrm{Eu} / \mathrm{Ba}, \mathrm{Gd} / \mathrm{Pr}$, and $\mathrm{Tb} / \mathrm{Nd}$ concentration ratios in extractant were constant at roughly 0.002 , 1.0 , and 0.04 , respectively, we prepared standard solution having the identical $\mathrm{Eu} / \mathrm{Ba}, \mathrm{Gd} / \mathrm{Pr}$, and $\mathrm{Tb} / \mathrm{Nd}$ ratios. Accordingly, it is presumed that there are no significant differences in the results.

\section{Handling of outliers}

The sequential extraction procedure provides a large variation in extracted data (e.g., Marin et al., 1997), and the determination of the mean and standard deviation $(\sigma)$ from the data is largely influenced by far outliers. For this reason, the median and median absolute deviation (MAD) were used to eliminate outliers (Miller and Miller, 2010). These statistical parameters are influenced by the data distribution. The MAD is defined by the following equation:

$$
\operatorname{MAD}=\operatorname{median}\left[\left|x_{i}-\operatorname{median}\left(x_{i}\right)\right|\right],
$$

where median $\left(x_{i}\right)$ means the median values of all data sets. 
Table 2. Concentrations $\left(\mathrm{mg} \mathrm{kg}^{-1}\right)$ and $95 \%$ confidence intervals for six elements in BCR-701 extracted using mechanical shaker operated at $30 \mathrm{rpm}$ and $80 \mathrm{rpm}$; significance probabilities ( $p$ ) of t-test

\begin{tabular}{|c|c|c|c|c|c|c|c|c|c|c|}
\hline Step 1 & $80 \mathrm{rpm}$ & $N$ & $30 \mathrm{rpm}$ & $N$ & $p^{a}$ & All & $N$ & $\operatorname{Ref}^{c}$ & $N^{\mathrm{c}}$ & $p^{b}$ \\
\hline $\mathrm{Cr}$ & $3.71 \pm 0.70$ & 3 & $2.81 \pm 0.72$ & 4 & 0.02 & $3.20 \pm 0.55$ & 7 & $2.26 \pm 0.16$ & 14 & 0.006 \\
\hline $\mathrm{Ni}$ & $17.1 \pm 0.5$ & 4 & $16.3 \pm 1.2$ & 4 & 0.125 & $16.7 \pm 0.6$ & 8 & $15.4 \pm 0.9$ & 13 & 0.014 \\
\hline $\mathrm{Cu}$ & $52.8 \pm 14.8$ & 4 & $49.4 \pm 9.5$ & 4 & 0.557 & $51.1 \pm 6.2$ & 8 & $49.3 \pm 1.7$ & 14 & 0.528 \\
\hline $\mathrm{Zn}$ & $213 \pm 12$ & 4 & $202 \pm 16$ & 4 & 0.134 & $208 \pm 9$ & 8 & $205 \pm 6$ & 14 & 0.576 \\
\hline $\mathrm{Cd}$ & $7.59 \pm 0.34$ & 4 & $7.44 \pm 0.49$ & 4 & 0.453 & $7.51 \pm 0.21$ & 8 & $7.34 \pm 0.35$ & 14 & 0.36 \\
\hline $\mathrm{Pb}$ & $3.85 \pm 0.41$ & 4 & $3.69 \pm 0.17$ & 4 & 0.28 & $3.77 \pm 0.17$ & 8 & $3.18 \pm 0.21$ & 14 & $<0.001$ \\
\hline \multicolumn{11}{|l|}{ Step 2} \\
\hline $\mathrm{Cr}$ & $46.9 \pm 1.8$ & 4 & $43.0 \pm 5.5$ & 4 & 0.077 & $45.0 \pm 2.7$ & 8 & $45.7 \pm 2.0$ & 12 & 0.626 \\
\hline $\mathrm{Ni}$ & $28.8 \pm 1.2$ & 4 & $27.8 \pm 2.8$ & 4 & 0.375 & $28.3 \pm 1.1$ & 8 & $26.6 \pm 1.3$ & 12 & 0.056 \\
\hline $\mathrm{Cu}$ & $109 \pm 9$ & 4 & $119 \pm 14$ & 4 & 0.098 & $114 \pm 8$ & 8 & $124 \pm 3$ & 14 & 0.003 \\
\hline $\mathrm{Zn}$ & $112 \pm 7$ & 4 & $109 \pm 13$ & 4 & 0.601 & $111 \pm 5$ & 8 & $114 \pm 5$ & 12 & 0.331 \\
\hline $\mathrm{Cd}$ & $3.69 \pm 0.09$ & 4 & $3.72 \pm 0.32$ & 3 & 0.77 & $3.70 \pm 0.08$ & 7 & $3.77 \pm 0.28$ & 14 & 0.627 \\
\hline $\mathrm{Pb}$ & $127 \pm 3$ & 4 & $126 \pm 4$ & 4 & 0.703 & $126 \pm 2$ & 8 & $126 \pm 3$ & 11 & 0.79 \\
\hline \multicolumn{11}{|l|}{ Step 3} \\
\hline $\mathrm{Cr}$ & $156 \pm 13$ & 4 & $155 \pm 2$ & 3 & 0.378 & $156 \pm 5$ & 7 & $143 \pm 7$ & 12 & 0.011 \\
\hline $\mathrm{Ni}$ & $16.1 \pm 1.5$ & 4 & $15.8 \pm 3.2$ & 4 & 0.764 & $16.0 \pm 1.2$ & 8 & $15.3 \pm 0.9$ & 13 & 0.332 \\
\hline $\mathrm{Cu}$ & $59.9 \pm 17.7$ & 4 & $48.8 \pm 37.0$ & 4 & 0.424 & $54.4 \pm 15$ & 8 & $55.2 \pm 4.0$ & 13 & 0.901 \\
\hline $\mathrm{Zn}$ & $47.2 \pm 7.8$ & 4 & $49.4 \pm 10.7$ & 3 & 0.823 & $48.3 \pm 4.6$ & 7 & $45.7 \pm 4.0$ & 11 & 0.652 \\
\hline $\mathrm{Cd}$ & $0.34 \pm 0.06$ & 4 & $0.28 \pm 0.10$ & 4 & 0.132 & $0.31 \pm 0.05$ & 8 & $0.27 \pm 0.06$ & 13 & 0.292 \\
\hline $\mathrm{Pb}$ & $7.30 \pm 1.09$ & 4 & $8.38 \pm 0.55$ & 3 & 0.115 & $7.76 \pm 0.71$ & 7 & $9.3 \pm 2.0$ & 13 & 0.131 \\
\hline \multicolumn{11}{|l|}{ Step 4} \\
\hline $\mathrm{Cr}$ & $109 \pm 7$ & 4 & $112 \pm 21$ & 3 & 0.553 & $110 \pm 6$ & 7 & $62.5 \pm 4.5$ & 13 & $<0.001$ \\
\hline $\mathrm{Ni}$ & $50.7 \pm 1.3$ & 4 & $49.8 \pm 5.5$ & 3 & 0.473 & $50.3 \pm 1.4$ & 7 & $41.4 \pm 2.7$ & 11 & $<0.001$ \\
\hline $\mathrm{Cu}$ & $49.6 \pm 8.2$ & 4 & $40.3 \pm 11.6$ & 3 & 0.058 & $45.6 \pm 6.2$ & 7 & $38.5 \pm 6.5$ & 14 & 0.145 \\
\hline $\mathrm{Zn}$ & $115 \pm 8$ & 4 & $113 \pm 26$ & 3 & 0.743 & $114 \pm 7$ & 7 & $95 \pm 8$ & 13 & 0.003 \\
\hline $\mathrm{Cd}$ & $0.19 \pm 0.10$ & 4 & $0.201 \pm 0.002$ & 4 & 0.282 & $0.20 \pm 0.03$ & 8 & $0.13 \pm 0.06$ & 9 & 0.045 \\
\hline $\mathrm{Pb}$ & $22.6 \pm 3.1$ & 4 & $20.7 \pm 7.4$ & 3 & 0.347 & $21.7 \pm 2.2$ & 7 & $11.0 \pm 3.0$ & 14 & $<0.001$ \\
\hline
\end{tabular}

$N$, number of determinations.

${ }^{a}$ Significance probabilities of t-test applied to extracted data obtained from different shaking speed.

${ }^{b}$ Significance probabilities of t-test applied to all extracted data and certified data (Sutherland, 2010).

${ }^{c}$ Certified data are cited from Sutherland (2010).

A bold-faced type means that the null hypothesis is rejected for $p=0.05$.

The MAD is calculated from the median of the absolute value of deviations of each value $\left(x_{i}\right)$ from the median values of all data sets $\left(\operatorname{median}\left(x_{i}\right)\right)$. The MAD is a robust estimator of $0.6745 \sigma$, estimated from distribution free data. On an empirical basis, median \pm 5 MAD is used as the threshold of the outliers.

\section{RESULTS}

Sequential extraction result of CRM-701

Shaking speed The BCR protocol recommends that the shaker is operated at $30 \pm 10 \mathrm{rpm}$ (e.g., Rauret et al., 1999). Quevauviller et al. (1997) proposed that the sample materials should be continually in suspension during the extraction. However, the Japanese geochemical reference materials in PFA tube with extractant were not adequately suspended at $30 \mathrm{rpm}$. By increasing the shaking speed gradually, it was determined that $80 \mathrm{rpm}$ is the minimum necessary speed to suspend samples in experimental solutions. Therefore, the speed of $80 \mathrm{rpm}$ was adopted in this study. In fact, however, the effect of shaking speed on the sequential extraction procedure remained unclear. In order to elucidate the effect of shaking speed, we extracted elements in CRM-701 using an end-overend mechanical shaker operated at speeds of 30 and 80 rpm. Table 2 summarizes the concentrations of $\mathrm{Cr}, \mathrm{Ni}$, $\mathrm{Cu}, \mathrm{Zn}, \mathrm{Cd}$, and $\mathrm{Pb}$ extracted at each step. The $t$-test was applied to compare the means of the two results. When 
Table 3. The concentrations in step 1 obtained from BCR extraction procedure

\begin{tabular}{|c|c|c|c|c|c|c|c|c|c|c|}
\hline \multirow[t]{2}{*}{ Elements } & \multirow[t]{2}{*}{ Unit } & \multirow[t]{2}{*}{ D.L. } & \multicolumn{2}{|l|}{ BCR701 } & \multicolumn{2}{|l|}{ JLk-1 } & \multicolumn{2}{|l|}{ JSd-1 } & \multicolumn{2}{|l|}{ JSd-2 } \\
\hline & & & Mean $\pm 95 \% *$ & $N$ & Mean $\pm 95 \% *$ & $N$ & Mean $\pm 95 \% *$ & $N$ & Mean $\pm 95 \% *$ & $N$ \\
\hline $\mathrm{Na}$ & $\mathrm{wt} \%$ & 0.00003 & $0.0057 \pm 0.0004$ & 7 & $0.0066 \pm 0.0003$ & 5 & $0.024 \pm 0.001$ & 7 & $0.027 \pm 0.002$ & 9 \\
\hline $\mathrm{Mg}$ & $\mathrm{wt} \%$ & 0.00003 & $0.081 \pm 0.001$ & 8 & $0.029 \pm 0.0006$ & 6 & $0.035 \pm 0.002$ & 8 & $0.056 \pm 0.005$ & 9 \\
\hline $\mathrm{Al}$ & $\mathrm{wt} \%$ & 0.0004 & $0.021 \pm 0.002$ & 8 & $0.022 \pm 0.002$ & 6 & $0.135 \pm 0.005$ & 8 & $0.10 \pm 0.01$ & 9 \\
\hline $\mathrm{P}$ & $\mathrm{mg} \mathrm{kg}^{-1}$ & 1 & $233 \pm 5$ & 7 & $4.3 \pm 1.2$ & 4 & $3.5 \pm 1.4$ & 6 & $21 \pm 3$ & 7 \\
\hline K & wt $\%$ & 0.0001 & $0.011 \pm 0.001$ & 7 & $0.026 \pm 0.001$ & 6 & $0.027 \pm 0.001$ & 6 & $0.027 \pm 0.004$ & 9 \\
\hline $\mathrm{Ca}$ & $\mathrm{wt} \%$ & 0.0001 & $0.752 \pm 0.006$ & 7 & $0.145 \pm 0.003$ & 7 & $0.110 \pm 0.001$ & 7 & $0.80 \pm 0.04$ & 9 \\
\hline $\mathrm{Sc}$ & $\mathrm{mg} \mathrm{kg}^{-1}$ & 0.05 & $0.17 \pm 0.03$ & 8 & $0.29 \pm 0.07$ & 6 & $0.47 \pm 0.14$ & 8 & $1.7 \pm 0.1$ & 7 \\
\hline $\mathrm{V}$ & $\mathrm{mg} \mathrm{kg}^{-1}$ & 1 & n.d. & - & n.d. & - & n.d. & - & n.d. & - \\
\hline $\mathrm{Cr}$ & $\mathrm{mg} \mathrm{kg}^{-1}$ & 0.07 & $3.2 \pm 0.55$ & 7 & $0.19 \pm 0.07$ & 6 & $0.31 \pm 0.05$ & 8 & $1.84 \pm 0.42$ & 9 \\
\hline $\mathrm{Mn}$ & $\mathrm{mg} \mathrm{kg}^{-1}$ & 0.02 & $201 \pm 2$ & 8 & $1260 \pm 10$ & 7 & $92 \pm 3$ & 7 & $151 \pm 7$ & 9 \\
\hline $\mathrm{Fe}$ & $\mathrm{wt} \%$ & 0.00004 & $0.021 \pm 0.004$ & 8 & $0.029 \pm 0.001$ & 5 & $0.032 \pm 0.003$ & 8 & $0.46 \pm 0.09$ & 9 \\
\hline Co & $\mathrm{mg} \mathrm{kg}^{-1}$ & 0.004 & $2.6 \pm 0.2$ & 8 & $1.17 \pm 0.08$ & 7 & $0.85 \pm 0.02$ & 7 & $12.4 \pm 0.4$ & 8 \\
\hline $\mathrm{Ni}$ & $\mathrm{mg} \mathrm{kg}^{-1}$ & 0.06 & $16.7 \pm 0.6$ & 8 & $1.13 \pm 0.08$ & 7 & $0.26 \pm 0.04$ & 7 & $10.1 \pm 0.3$ & 9 \\
\hline $\mathrm{Cu}$ & $\mathrm{mg} \mathrm{kg}^{-1}$ & 0.2 & $51.1 \pm 6.2$ & 8 & $5.13 \pm 0.22$ & 7 & $1.32 \pm 0.12$ & 8 & $176 \pm 17$ & 9 \\
\hline $\mathrm{Zn}$ & $\mathrm{mg} \mathrm{kg}^{-1}$ & 0.1 & $208 \pm 9$ & 8 & $5.69 \pm 0.3$ & 5 & $11.8 \pm 0.7$ & 8 & $434 \pm 65$ & 9 \\
\hline $\mathrm{Rb}$ & $\mathrm{mg} \mathrm{kg}^{-1}$ & 0.002 & $0.38 \pm 0.01$ & 8 & $0.63 \pm 0.04$ & 7 & $0.38 \pm 0.03$ & 8 & $0.45 \pm 0.07$ & 9 \\
\hline $\mathrm{Sr}$ & $\mathrm{mg} \mathrm{kg}^{-1}$ & 0.01 & $48.9 \pm 0.3$ & 7 & $7.84 \pm 0.09$ & 7 & $14.2 \pm 0.4$ & 7 & $15.3 \pm 0.5$ & 9 \\
\hline $\mathrm{Y}$ & $\mathrm{mg} \mathrm{kg}^{-1}$ & 0.002 & $0.44 \pm 0.07$ & 8 & $1.9 \pm 0.3$ & 6 & $0.65 \pm 0.03$ & 7 & $1.10 \pm 0.07$ & 9 \\
\hline Mo & $\mathrm{mg} \mathrm{kg}^{-1}$ & 0.004 & $0.035 \pm 0.009$ & 8 & $0.009 \pm 0.005$ & 5 & $0.005 \pm 0.002$ & 5 & $0.076 \pm 0.021$ & 9 \\
\hline $\mathrm{Cd}$ & $\mathrm{mg} \mathrm{kg}^{-1}$ & 0.002 & $7.51 \pm 0.21$ & 8 & $0.34 \pm 0.01$ & 7 & $0.047 \pm 0.002$ & 7 & $1.29 \pm 0.07$ & 9 \\
\hline $\mathrm{Cs}$ & $\mathrm{mg} \mathrm{kg}^{-1}$ & 0.0003 & $0.0053 \pm 0.0006$ & 8 & $0.0055 \pm 0.0006$ & 7 & $0.0015 \pm 0.0011$ & 6 & $0.0023 \pm 0.0008$ & 7 \\
\hline $\mathrm{Ba}$ & $\mathrm{mg} \mathrm{kg}^{-1}$ & 0.08 & $22.2 \pm 0.6$ & 8 & $22.9 \pm 0.9$ & 7 & $45.3 \pm 0.9$ & 7 & $107 \pm 8$ & 9 \\
\hline $\mathrm{La}$ & $\mathrm{mg} \mathrm{kg}^{-1}$ & 0.003 & $0.32 \pm 0.04$ & 8 & $1.1 \pm 0.1$ & 6 & $1.4 \pm 0.1$ & 8 & $0.63 \pm 0.05$ & 9 \\
\hline $\mathrm{Ce}$ & $\mathrm{mg} \mathrm{kg}^{-1}$ & 0.005 & $0.68 \pm 0.1$ & 8 & $2.6 \pm 0.4$ & 6 & $1.6 \pm 0.1$ & 7 & $1.1 \pm 0.1$ & 9 \\
\hline $\operatorname{Pr}$ & $\mathrm{mg} \mathrm{kg}^{-1}$ & 0.0006 & $0.072 \pm 0.011$ & 8 & $0.29 \pm 0.04$ & 6 & $0.29 \pm 0.01$ & 8 & $0.17 \pm 0.01$ & 9 \\
\hline $\mathrm{Nd}$ & $\mathrm{mg} \mathrm{kg}^{-1}$ & 0.003 & $0.31 \pm 0.05$ & 8 & $1.2 \pm 0.2$ & 6 & $1.1 \pm 0.1$ & 8 & $0.72 \pm 0.06$ & 9 \\
\hline $\mathrm{Sm}$ & $\mathrm{mg} \mathrm{kg}^{-1}$ & 0.001 & $0.065 \pm 0.011$ & 8 & $0.32 \pm 0.05$ & 6 & $0.19 \pm 0.01$ & 8 & $0.17 \pm 0.01$ & 9 \\
\hline $\mathrm{Eu}$ & $\mathrm{mg} \mathrm{kg}^{-1}$ & 0.0003 & $0.013 \pm 0.002$ & 8 & $0.050 \pm 0.008$ & 6 & $0.050 \pm 0.001$ & 6 & $0.052 \pm 0.005$ & 9 \\
\hline $\mathrm{Gd}$ & $\mathrm{mg} \mathrm{kg}^{-1}$ & 0.001 & $0.074 \pm 0.014$ & 8 & $0.35 \pm 0.05$ & 6 & $0.17 \pm 0.01$ & 8 & $0.19 \pm 0.01$ & 9 \\
\hline $\mathrm{Tb}$ & $\mathrm{mg} \mathrm{kg}^{-1}$ & 0.0002 & $0.010 \pm 0.001$ & 8 & $0.053 \pm 0.008$ & 6 & $0.021 \pm 0.001$ & 8 & $0.028 \pm 0.002$ & 9 \\
\hline Dy & $\mathrm{mg} \mathrm{kg}^{-1}$ & 0.0008 & $0.058 \pm 0.012$ & 8 & $0.31 \pm 0.04$ & 6 & $0.11 \pm 0.01$ & 8 & $0.17 \pm 0.01$ & 9 \\
\hline Ho & $\mathrm{mg} \mathrm{kg}^{-1}$ & 0.0002 & $0.012 \pm 0.002$ & 8 & $0.058 \pm 0.009$ & 6 & $0.021 \pm 0.001$ & 8 & $0.036 \pm 0.002$ & 9 \\
\hline Er & $\mathrm{mg} \mathrm{kg}^{-1}$ & 0.0009 & $0.033 \pm 0.007$ & 8 & $0.16 \pm 0.03$ & 6 & $0.057 \pm 0.002$ & 8 & $0.10 \pm 0.01$ & 9 \\
\hline $\mathrm{Tm}$ & $\mathrm{mg} \mathrm{kg}^{-1}$ & 0.0001 & $0.0046 \pm 0.0008$ & 8 & $0.022 \pm 0.004$ & 6 & $0.0078 \pm 0.0003$ & 8 & $0.015 \pm 0.001$ & 9 \\
\hline $\mathrm{Yb}$ & $\mathrm{mg} \mathrm{kg}^{-1}$ & 0.001 & $0.031 \pm 0.006$ & 8 & $0.13 \pm 0.02$ & 6 & $0.047 \pm 0.002$ & 8 & $0.095 \pm 0.007$ & 9 \\
\hline $\mathrm{Lu}$ & $\mathrm{mg} \mathrm{kg}^{-1}$ & 0.0001 & $0.0047 \pm 0.0009$ & 8 & $0.019 \pm 0.003$ & 6 & $0.0071 \pm 0.0004$ & 8 & $0.014 \pm 0.001$ & 9 \\
\hline $\mathrm{Pb}$ & $\mathrm{mg} \mathrm{kg}^{-1}$ & 0.05 & $3.77 \pm 0.17$ & 8 & $0.98 \pm 0.09$ & 7 & $0.077 \pm 0.020$ & 6 & $10.9 \pm 1.4$ & 9 \\
\hline $\mathrm{Bi}$ & $\mathrm{mg} \mathrm{kg}^{-1}$ & 0.006 & $0.010 \pm 0.002$ & 8 & $0.014 \pm 0.004$ & 7 & $0.0069 \pm 0.0027$ & 7 & $0.014 \pm 0.002$ & 9 \\
\hline
\end{tabular}

the probability obtained by $t$-test was less than 0.05 , we could conclude that the two means differed significantly. The results of the statistical test suggest that only concentrations of $\mathrm{Cr}$ extracted in step 1 differed significantly (Table 2). Although all elements, except for $\mathrm{Cr}, \mathrm{Ni}, \mathrm{Cu}$, $\mathrm{Zn}, \mathrm{Cd}$, and $\mathrm{Pb}$, are not certified for CRM-701; Mo, Y, and the lanthanides (Ln) also showed the same results as $\mathrm{Cr}$ extracted in step 1. However, the ratios of these elemental concentrations extracted in step 1 to bulk concentrations were less than $1.5 \%$. For example, the percentages of $\mathrm{Cr}$ concentrations in step 1 to bulk composition at the speeds of 30 and $80 \mathrm{rpm}$ were $1.2 \%$ and $0.9 \%$, respectively. Therefore, although the concentrations of several elements extracted in step 1 varied significantly by shaking speed, we judged that it was of no major consequence for discussion of the extraction percentage.

Precision and accuracy of extracted data Table 2 also includes the results of repeated analyses of the BCR-701 reference samples for the certified six elements calculated from all data. The relative standard deviations (RSDs) for the six elements extracted in these three steps were less than $\pm 10 \%$ in most cases; $95 \%$ confidential intervals obtained in this study were comparable to the certified values (Table 2). However, the worse RSDs were determined for $\mathrm{Cr}$ extracted in step 1; for $\mathrm{Cu}$ in steps 1, 3, and 4 ; and for $\mathrm{Cd}$ in steps 3 and $4(15-33 \%)$. 
Table 3. (continued)

\begin{tabular}{|c|c|c|c|c|c|c|c|c|c|c|c|}
\hline \multirow[t]{2}{*}{ Elements } & \multirow[t]{2}{*}{ Unit } & \multicolumn{2}{|l|}{ JSd-3 } & \multicolumn{2}{|l|}{ JSd-4 } & \multicolumn{2}{|l|}{ JMs-1 } & \multicolumn{2}{|l|}{ JMs-2 } & \multicolumn{2}{|l|}{ JSO-1 } \\
\hline & & Mean $\pm 95 \% *$ & $N$ & Mean $\pm 95 \% *$ & $N$ & Mean $\pm 95 \% *$ & $N$ & Mean $\pm 95 \% *$ & $N$ & Mean $\pm 95 \% *$ & $N$ \\
\hline $\mathrm{Na}$ & wt $\%$ & $0.003 \pm 0.001$ & 6 & $0.75 \pm 0.05$ & 7 & $1.86 \pm 0.05$ & 6 & $2.69 \pm 0.07$ & 6 & $0.0079 \pm 0.0005$ & 8 \\
\hline $\mathrm{Mg}$ & $\mathrm{wt} \%$ & $0.010 \pm<0.001$ & 4 & $0.65 \pm 0.02$ & 8 & $0.36 \pm 0.01$ & 8 & $0.47 \pm 0.01$ & 7 & $0.029 \pm 0.001$ & 8 \\
\hline $\mathrm{Al}$ & $\mathrm{wt} \%$ & $0.110 \pm 0.005$ & 7 & $0.069 \pm 0.01$ & 8 & $0.080 \pm 0.001$ & 5 & $0.031 \pm 0.002$ & 7 & $0.22 \pm 0.02$ & 9 \\
\hline$P$ & $\mathrm{mg} \mathrm{kg}^{-1}$ & $2.9 \pm 0.3$ & 5 & $8.1 \pm 2.6$ & 8 & $6.0 \pm 0.8$ & 6 & $73 \pm 16$ & 8 & $4.5 \pm 1.7$ & 8 \\
\hline K & wt $\%$ & $0.026 \pm 0.001$ & 5 & $0.118 \pm 0.008$ & 8 & $0.22 \pm 0.02$ & 7 & $0.22 \pm 0.03$ & 8 & $0.022 \pm 0.002$ & 8 \\
\hline $\mathrm{Ca}$ & $\mathrm{wt} \%$ & $0.062 \pm 0.001$ & 7 & $2.57 \pm 0.04$ & 8 & $0.61 \pm 0.02$ & 8 & $1.14 \pm 0.04$ & 8 & $0.520 \pm 0.002$ & 7 \\
\hline $\mathrm{Sc}$ & $\mathrm{mg} \mathrm{kg}^{-1}$ & $0.28 \pm 0.04$ & 7 & $0.73 \pm 0.15$ & 9 & $0.60 \pm 0.01$ & 5 & $0.80 \pm 0.07$ & 7 & $0.70 \pm 0.06$ & 6 \\
\hline $\mathrm{V}$ & $\mathrm{mg} \mathrm{kg}^{-1}$ & n.d. & - & $1.3 \pm 0.4$ & 3 & $1.9 \pm 0.7$ & 4 & $1.5 \pm 0.6$ & 3 & n.d. & - \\
\hline $\mathrm{Cr}$ & $\mathrm{mg} \mathrm{kg}^{-1}$ & $0.217 \pm 0.05$ & 6 & $50 \pm 12$ & 9 & $3.05 \pm 0.51$ & 8 & $1.14 \pm 0.09$ & 7 & $0.35 \pm 0.1$ & 8 \\
\hline Mn & $\mathrm{mg} \mathrm{kg}^{-1}$ & $190 \pm 10$ & 7 & $179 \pm 10$ & 9 & $159 \pm 5$ & 8 & $370 \pm 100$ & 6 & $140 \pm 13$ & 9 \\
\hline $\mathrm{Fe}$ & $\mathrm{wt} \%$ & $0.0023 \pm 0.0002$ & 6 & $0.037 \pm 0.008$ & 9 & $0.034 \pm 0.004$ & 8 & $0.0004 \pm 0.0001$ & 7 & $0.008 \pm 0.002$ & 9 \\
\hline Co & $\mathrm{mg} \mathrm{kg}^{-1}$ & $2.5 \pm 0.3$ & 8 & $2.9 \pm 0.2$ & 9 & $2.2 \pm 0.2$ & 8 & $0.74 \pm 0.31$ & 5 & $1.4 \pm 0.3$ & 8 \\
\hline $\mathrm{Ni}$ & $\mathrm{mg} \mathrm{kg}^{-1}$ & $0.69 \pm 0.06$ & 6 & $23.2 \pm 1.9$ & 8 & $4.47 \pm 0.35$ & 8 & $51.3 \pm 9.9$ & 8 & $0.70 \pm 0.03$ & 6 \\
\hline $\mathrm{Cu}$ & $\mathrm{mg} \mathrm{kg}^{-1}$ & $84.6 \pm 3.4$ & 6 & $55.3 \pm 8.9$ & 9 & $5.44 \pm 0.87$ & 8 & $8.73 \pm 0.99$ & 6 & $1.14 \pm 0.33$ & 8 \\
\hline $\mathrm{Zn}$ & $\mathrm{mg} \mathrm{kg}^{-1}$ & $11.4 \pm 1.5$ & 7 & $458 \pm 61$ & 9 & $80.7 \pm 4.0$ & 8 & $6.3 \pm 1.1$ & 7 & $4.8 \pm 1.0$ & 8 \\
\hline $\mathrm{Rb}$ & $\mathrm{mg} \mathrm{kg}^{-1}$ & $2.48 \pm 0.09$ & 7 & $0.89 \pm 0.03$ & 8 & $1.10 \pm 0.05$ & 8 & $0.48 \pm 0.05$ & 7 & $0.79 \pm 0.07$ & 8 \\
\hline $\mathrm{Sr}$ & $\mathrm{mg} \mathrm{kg}^{-1}$ & $3.6 \pm 0.1$ & 7 & $81.4 \pm 0.8$ & 8 & $53.1 \pm 1$ & 8 & $130 \pm 4$ & 8 & $84 \pm 1$ & 9 \\
\hline Y & $\mathrm{mg} \mathrm{kg}^{-1}$ & $1.1 \pm 0.1$ & 8 & $1.4 \pm 0.2$ & 9 & $1.5 \pm 0.2$ & 8 & $11 \pm 2$ & 8 & $0.34 \pm 0.07$ & 8 \\
\hline Mo & $\mathrm{mg} \mathrm{kg}^{-1}$ & $0.005 \pm 0.002$ & 3 & $0.016 \pm 0.006$ & 8 & $0.012 \pm 0.007$ & 6 & $0.024 \pm 0.006$ & 7 & $0.008 \pm 0.006$ & 5 \\
\hline $\mathrm{Cd}$ & $\mathrm{mg} \mathrm{kg}^{-1}$ & $0.54 \pm 0.02$ & 7 & $4.36 \pm 0.38$ & 9 & $0.78 \pm 0.03$ & 8 & $0.088 \pm 0.013$ & 7 & $0.067 \pm 0.009$ & 7 \\
\hline Cs & $\mathrm{mg} \mathrm{kg}^{-1}$ & $0.070 \pm 0.004$ & 8 & $0.0081 \pm 0.0006$ & 8 & $0.0064 \pm 0.0013$ & 6 & $0.0017 \pm 0.0009$ & 6 & $0.018 \pm 0.004$ & 7 \\
\hline $\mathrm{Ba}$ & $\mathrm{mg} \mathrm{kg}^{-1}$ & $24.6 \pm 0.6$ & 7 & $12.9 \pm 1.3$ & 8 & $4.5 \pm 0.2$ & 8 & $1.1 \pm 0.1$ & 8 & $80 \pm 3.5$ & 8 \\
\hline $\mathrm{La}$ & $\mathrm{mg} \mathrm{kg}^{-1}$ & $0.73 \pm 0.07$ & 8 & $0.88 \pm 0.14$ & 9 & $1.0 \pm 0.1$ & 8 & $3.2 \pm 0.5$ & 7 & $0.17 \pm 0.03$ & 8 \\
\hline $\mathrm{Ce}$ & $\mathrm{mg} \mathrm{kg}^{-1}$ & $1.1 \pm 0.1$ & 8 & $1.3 \pm 0.2$ & 9 & $2.1 \pm 0.3$ & 8 & $0.29 \pm 0.09$ & 7 & $0.15 \pm 0.04$ & 8 \\
\hline $\operatorname{Pr}$ & $\mathrm{mg} \mathrm{kg}^{-1}$ & $0.16 \pm 0.02$ & 8 & $0.18 \pm 0.03$ & 9 & $0.24 \pm 0.03$ & 8 & $0.70 \pm 0.06$ & 6 & $0.037 \pm 0.007$ & 8 \\
\hline $\mathrm{Nd}$ & $\mathrm{mg} \mathrm{kg}^{-1}$ & $0.64 \pm 0.08$ & 8 & $0.80 \pm 0.14$ & 9 & $1.0 \pm 0.1$ & 8 & $3.9 \pm 0.1$ & 5 & $0.18 \pm 0.04$ & 8 \\
\hline $\mathrm{Sm}$ & $\mathrm{mg} \mathrm{kg}^{-1}$ & $0.14 \pm 0.02$ & 8 & $0.18 \pm 0.03$ & 9 & $0.23 \pm 0.03$ & 8 & $0.86 \pm 0.08$ & 6 & $0.040 \pm 0.009$ & 8 \\
\hline $\mathrm{Eu}$ & $\mathrm{mg} \mathrm{kg}^{-1}$ & $0.037 \pm 0.005$ & 8 & $0.046 \pm 0.009$ & 9 & $0.06 \pm 0.01$ & 8 & $0.22 \pm 0.02$ & 6 & $0.016 \pm 0.002$ & 8 \\
\hline Gd & $\mathrm{mg} \mathrm{kg}^{-1}$ & $0.17 \pm 0.02$ & 8 & $0.21 \pm 0.04$ & 9 & $0.27 \pm 0.03$ & 8 & $1.3 \pm 0.1$ & 6 & $0.044 \pm 0.006$ & 7 \\
\hline $\mathrm{Tb}$ & $\mathrm{mg} \mathrm{kg}^{-1}$ & $0.025 \pm 0.004$ & 8 & $0.029 \pm 0.005$ & 9 & $0.039 \pm 0.005$ & 8 & $0.165 \pm 0.001$ & 5 & $0.0068 \pm 0.0011$ & 6 \\
\hline Dy & $\mathrm{mg} \mathrm{kg}^{-1}$ & $0.16 \pm 0.02$ & 8 & $0.17 \pm 0.03$ & 9 & $0.22 \pm 0.03$ & 8 & $1.07 \pm 0.02$ & 5 & $0.041 \pm 0.009$ & 8 \\
\hline Но & $\mathrm{mg} \mathrm{kg}^{-1}$ & $0.031 \pm 0.003$ & 7 & $0.035 \pm 0.006$ & 9 & $0.044 \pm 0.005$ & 8 & $0.27 \pm 0.01$ & 5 & $0.0088 \pm 0.001$ & 7 \\
\hline $\mathrm{Er}$ & $\mathrm{mg} \mathrm{kg}^{-1}$ & $0.090 \pm 0.010$ & 8 & $0.10 \pm 0.02$ & 9 & $0.11 \pm 0.01$ & 8 & $0.81 \pm 0.01$ & 5 & $0.024 \pm 0.004$ & 7 \\
\hline $\mathrm{Tm}$ & $\mathrm{mg} \mathrm{kg}^{-1}$ & $0.012 \pm 0.001$ & 7 & $0.013 \pm 0.002$ & 9 & $0.015 \pm 0.002$ & 8 & $0.10 \pm 0.01$ & 6 & $0.0036 \pm 0.0008$ & 7 \\
\hline $\mathrm{Yb}$ & $\mathrm{mg} \mathrm{kg}^{-1}$ & $0.076 \pm 0.009$ & 8 & $0.082 \pm 0.014$ & 9 & $0.091 \pm 0.013$ & 8 & $0.66 \pm 0.01$ & 5 & $0.025 \pm 0.005$ & 8 \\
\hline $\mathrm{Lu}$ & $\mathrm{mg} \mathrm{kg}^{-1}$ & $0.011 \pm 0.002$ & 8 & $0.014 \pm 0.002$ & 9 & $0.014 \pm 0.002$ & 8 & $0.125 \pm 0.004$ & 5 & $0.004 \pm 0.0006$ & 7 \\
\hline $\mathrm{Pb}$ & $\mathrm{mg} \mathrm{kg}^{-1}$ & $2.49 \pm 0.24$ & 8 & $2.60 \pm 0.26$ & 9 & $1.09 \pm 0.12$ & 8 & $<0.09$ & 2 & $0.082 \pm 0.004$ & 3 \\
\hline $\mathrm{Bi}$ & $\mathrm{mg} \mathrm{kg}^{-1}$ & $0.036 \pm 0.003$ & 7 & $0.010 \pm 0.002$ & 8 & $0.0093 \pm 0.0021$ & 7 & $0.0061 \pm 0.0016$ & 6 & $0.0060 \pm 0.0019$ & 7 \\
\hline
\end{tabular}

$N$, number of determinations; D.L., detection limit; n.d., not determinated; *95\% confidence interval.

Next, the $t$-test was applied to confirm how our data compared to the certified values for respective elements and respective steps. The statistical results suggest that the concentrations of $\mathrm{Cr}, \mathrm{Ni}$, and $\mathrm{Pb}$ extracted in step 1 and those of $\mathrm{Cr}$ in step 3 were significantly higher than the certified values. This occurred because the water used for washing the residue was added into the extracted solution without discarding. Meanwhile, the concentration of $\mathrm{Cu}$ obtained at step 2 was significantly lower than the certified value. The concentrations of $\mathrm{Cr}, \mathrm{Ni}, \mathrm{Cu}, \mathrm{Zn}$, and $\mathrm{Pb}$ in step 4 were a reasonable amount higher than the certified values because the residue from step 3 was di- gested not by HF but by aqua regia in the BCR protocol. Accordingly, we can conclude that the obtained data are consistent with the certified values.

\section{Precision and recovery of sequential extraction results of Japanese geochemical reference materials}

The sequential extraction results of the eight geochemical reference samples and CRM-701 are presented in Tables 3-6. Figure 2 shows the RSDs obtained from repeated measurements for the 38 elements in each step. The RSDs for most elements were less than $30 \%$ for steps 1 and 3 and less than $20 \%$ for steps 2 and 4 . The 
Table 4. The concentrations in step 2 obtained from BCR extraction procedure

\begin{tabular}{|c|c|c|c|c|c|c|c|c|c|c|}
\hline \multirow[t]{2}{*}{ Elements } & \multirow[t]{2}{*}{ Unit } & \multirow[t]{2}{*}{ D.L. } & \multicolumn{2}{|l|}{ BCR701 } & \multicolumn{2}{|l|}{ JLk-1 } & \multicolumn{2}{|l|}{ JSd-1 } & \multicolumn{2}{|l|}{$\mathrm{JSd}-2$} \\
\hline & & & Mean $\pm 95 \% *$ & $N$ & Mean $\pm 95 \% *$ & $N$ & Mean $\pm 95 \% *$ & $N$ & Mean $\pm 95 \% *$ & $N$ \\
\hline $\mathrm{Na}$ & $\mathrm{wt} \%$ & 0.0001 & $0.0009 \pm 0.0002$ & 6 & $0.0013 \pm 0.0003$ & 6 & $0.0079 \pm 0.0004$ & 5 & $0.023 \pm 0.004$ & 9 \\
\hline $\mathrm{Mg}$ & $\mathrm{wt} \%$ & 0.00003 & $0.077 \pm 0.005$ & 8 & $0.067 \pm 0.007$ & 6 & $0.082 \pm 0.007$ & 7 & $0.13 \pm 0.01$ & 9 \\
\hline $\mathrm{Al}$ & $\mathrm{wt} \%$ & 0.0003 & $0.38 \pm 0.01$ & 8 & $0.37 \pm 0.01$ & 5 & $0.38 \pm 0.01$ & 7 & $0.41 \pm 0.01$ & 9 \\
\hline$P$ & $\mathrm{mg} \mathrm{kg}^{-1}$ & 1 & $1540 \pm 110$ & 7 & $450 \pm 100$ & 5 & $293 \pm 12$ & 5 & $261 \pm 41$ & 5 \\
\hline K & $\mathrm{wt} \%$ & 0.0001 & $0.016 \pm 0.002$ & 8 & $0.027 \pm 0.001$ & 5 & $0.035 \pm 0.001$ & 5 & $0.049 \pm 0.005$ & 8 \\
\hline $\mathrm{Ca}$ & wt $\%$ & 0.0001 & $0.189 \pm 0.005$ & 8 & $0.076 \pm 0.003$ & 6 & $0.094 \pm 0.002$ & 7 & $0.52 \pm 0.04$ & 9 \\
\hline Sc & $\mathrm{mg} \mathrm{kg}^{-1}$ & 0.09 & $0.27 \pm 0.24$ & 5 & $0.86 \pm 0.34$ & 6 & $0.65 \pm 0.41$ & 7 & $2.9 \pm 0.6$ & 8 \\
\hline $\mathrm{V}$ & $\mathrm{mg} \mathrm{kg}^{-1}$ & 0.8 & $12 \pm 1$ & 5 & $19 \pm 2$ & 5 & $8.4 \pm 2.0$ & 6 & $8.3 \pm 1.8$ & 7 \\
\hline $\mathrm{Cr}$ & $\mathrm{mg} \mathrm{kg}^{-1}$ & 0.7 & $45 \pm 2.7$ & 8 & $4.8 \pm 1.9$ & 6 & $1.39 \pm 0.55$ & 5 & $11.7 \pm 0.2$ & 5 \\
\hline $\mathrm{Mn}$ & $\mathrm{mg} \mathrm{kg}^{-1}$ & 0.03 & $136 \pm 4$ & 7 & $361 \pm 11$ & 7 & $99 \pm 2$ & 6 & $174 \pm 6$ & 8 \\
\hline $\mathrm{Fe}$ & $\mathrm{wt} \%$ & 0.00002 & $0.82 \pm 0.02$ & 7 & $1.44 \pm 0.12$ & 6 & $0.41 \pm 0.02$ & 6 & $1.83 \pm 0.07$ & 8 \\
\hline Co & $\mathrm{mg} \mathrm{kg}^{-1}$ & 0.007 & $3.2 \pm 0.1$ & 8 & $4.2 \pm 0.2$ & 6 & $1.55 \pm 0.05$ & 7 & $15.8 \pm 0.5$ & 9 \\
\hline $\mathrm{Ni}$ & $\mathrm{mg} \mathrm{kg}^{-1}$ & 0.08 & $28.3 \pm 1.1$ & 8 & $4.08 \pm 0.12$ & 5 & $0.94 \pm 0.22$ & 7 & $34.7 \pm 1.4$ & 9 \\
\hline $\mathrm{Cu}$ & $\mathrm{mg} \mathrm{kg}^{-1}$ & 0.2 & $114 \pm 8$ & 8 & $15.7 \pm 0.7$ & 6 & $7.58 \pm 0.74$ & 7 & $237 \pm 22$ & 9 \\
\hline $\mathrm{Zn}$ & $\mathrm{mg} \mathrm{kg}^{-1}$ & 0.3 & $111 \pm 5$ & 8 & $26.4 \pm 1.3$ & 5 & $16.9 \pm 1.3$ & 6 & $1325 \pm 91$ & 9 \\
\hline $\mathrm{Rb}$ & $\mathrm{mg} \mathrm{kg}^{-1}$ & 0.01 & $2.2 \pm 0.1$ & 8 & $4.0 \pm 0.2$ & 6 & $3.5 \pm 0.2$ & 6 & $2.3 \pm 0.1$ & 8 \\
\hline $\mathrm{Sr}$ & $\mathrm{mg} \mathrm{kg}^{-1}$ & 0.02 & $14.7 \pm 0.5$ & 7 & $7.9 \pm 0.3$ & 6 & $4.85 \pm 0.08$ & 7 & $17.9 \pm 0.8$ & 9 \\
\hline $\mathrm{Y}$ & $\mathrm{mg} \mathrm{kg}^{-1}$ & 0.005 & $7.6 \pm 0.3$ & 8 & $17.5 \pm 0.4$ & 7 & $2.03 \pm 0.05$ & 7 & $2.38 \pm 0.07$ & 9 \\
\hline Mo & $\mathrm{mg} \mathrm{kg}^{-1}$ & 0.01 & $0.13 \pm 0.03$ & 8 & $0.074 \pm 0.024$ & 7 & $0.018 \pm 0.007$ & 3 & $0.66 \pm 0.06$ & 9 \\
\hline $\mathrm{Cd}$ & $\mathrm{mg} \mathrm{kg}^{-1}$ & 0.003 & $3.66 \pm 0.13$ & 8 & $0.140 \pm 0.012$ & 6 & $0.035 \pm 0.007$ & 6 & $1.03 \pm 0.03$ & 8 \\
\hline Cs & $\mathrm{mg} \mathrm{kg}^{-1}$ & 0.0008 & $0.35 \pm 0.01$ & 8 & $0.35 \pm 0.02$ & 7 & $0.11 \pm 0.01$ & 6 & $0.121 \pm 0.004$ & 7 \\
\hline $\mathrm{Ba}$ & $\mathrm{mg} \mathrm{kg}^{-1}$ & 0.07 & $118 \pm 3$ & 8 & $121 \pm 2$ & 6 & $53 \pm 1$ & 8 & $718 \pm 16$ & 9 \\
\hline $\mathrm{La}$ & $\mathrm{mg} \mathrm{kg}^{-1}$ & 0.008 & $6.5 \pm 0.2$ & 8 & $14.6 \pm 0.3$ & 7 & $2.80 \pm 0.06$ & 7 & $1.61 \pm 0.06$ & 9 \\
\hline $\mathrm{Ce}$ & $\mathrm{mg} \mathrm{kg}^{-1}$ & 0.02 & $17.5 \pm 0.7$ & 8 & $35 \pm 1$ & 7 & $5.9 \pm 0.3$ & 7 & $3.1 \pm 0.1$ & 9 \\
\hline $\operatorname{Pr}$ & $\mathrm{mg} \mathrm{kg}^{-1}$ & 0.001 & $1.69 \pm 0.03$ & 8 & $3.81 \pm 0.09$ & 7 & $0.74 \pm 0.01$ & 7 & $0.44 \pm 0.01$ & 9 \\
\hline $\mathrm{Nd}$ & $\mathrm{mg} \mathrm{kg}^{-1}$ & 0.01 & $6.8 \pm 0.2$ & 8 & $15.0 \pm 0.5$ & 7 & $2.93 \pm 0.05$ & 7 & $1.84 \pm 0.06$ & 9 \\
\hline $\mathrm{Sm}$ & $\mathrm{mg} \mathrm{kg}^{-1}$ & 0.004 & $1.55 \pm 0.04$ & 8 & $3.64 \pm 0.14$ & 7 & $0.59 \pm 0.02$ & 7 & $0.45 \pm 0.02$ & 9 \\
\hline $\mathrm{Eu}$ & $\mathrm{mg} \mathrm{kg}^{-1}$ & 0.001 & $0.24 \pm 0.01$ & 8 & $0.55 \pm 0.02$ & 7 & $0.11 \pm 0.01$ & 7 & $0.15 \pm 0.01$ & 9 \\
\hline $\mathrm{Gd}$ & $\mathrm{mg} \mathrm{kg}^{-1}$ & 0.005 & $1.59 \pm 0.04$ & 8 & $3.69 \pm 0.08$ & 7 & $0.54 \pm 0.02$ & 7 & $0.47 \pm 0.02$ & 9 \\
\hline $\mathrm{Tb}$ & $\mathrm{mg} \mathrm{kg}^{-1}$ & 0.0005 & $0.24 \pm 0.01$ & 8 & $0.56 \pm 0.01$ & 7 & $0.072 \pm 0.003$ & 7 & $0.071 \pm 0.002$ & 9 \\
\hline Dy & $\mathrm{mg} \mathrm{kg}^{-1}$ & 0.002 & $1.48 \pm 0.03$ & 8 & $3.25 \pm 0.07$ & 7 & $0.39 \pm 0.01$ & 7 & $0.45 \pm 0.01$ & 9 \\
\hline Ho & $\mathrm{mg} \mathrm{kg}^{-1}$ & 0.0006 & $0.274 \pm 0.005$ & 8 & $0.61 \pm 0.01$ & 7 & $0.075 \pm 0.005$ & 7 & $0.090 \pm 0.001$ & 9 \\
\hline Er & $\mathrm{mg} \mathrm{kg}^{-1}$ & 0.001 & $0.75 \pm 0.01$ & 8 & $1.64 \pm 0.03$ & 7 & $0.20 \pm 0.01$ & 7 & $0.26 \pm 0.01$ & 9 \\
\hline $\mathrm{Tm}$ & $\mathrm{mg} \mathrm{kg}^{-1}$ & 0.0005 & $0.099 \pm 0.003$ & 8 & $0.22 \pm 0.01$ & 7 & $0.026 \pm 0.005$ & 8 & $0.035 \pm 0.002$ & 9 \\
\hline $\mathrm{Yb}$ & $\mathrm{mg} \mathrm{kg}^{-1}$ & 0.005 & $0.63 \pm 0.01$ & 8 & $1.33 \pm 0.03$ & 7 & $0.17 \pm 0.01$ & 7 & $0.22 \pm 0.01$ & 9 \\
\hline $\mathrm{Lu}$ & $\mathrm{mg} \mathrm{kg}^{-1}$ & 0.0003 & $0.081 \pm 0.003$ & 8 & $0.19 \pm 0.01$ & 7 & $0.023 \pm 0.001$ & 5 & $0.030 \pm 0.001$ & 9 \\
\hline $\mathrm{Pb}$ & $\mathrm{mg} \mathrm{kg}^{-1}$ & 0.1 & $126 \pm 2$ & 8 & $32.3 \pm 1.4$ & 7 & $4.56 \pm 0.29$ & 6 & $104 \pm 5$ & 9 \\
\hline $\mathrm{Bi}$ & $\mathrm{mg} \mathrm{kg}^{-1}$ & 0.006 & $0.91 \pm 0.08$ & 8 & $0.69 \pm 0.02$ & 7 & $0.034 \pm 0.011$ & 7 & $0.27 \pm 0.02$ & 9 \\
\hline
\end{tabular}

worse coefficients of variance were determined for $\mathrm{P}, \mathrm{V}$, and $\mathrm{Cr}$ in steps 1,2, and 3; for Sc in steps 1 and 2; for $\mathrm{Fe}$ and $\mathrm{Bi}$ in steps 1 and 3; for $\mathrm{Na}$ and $\mathrm{Mo}$ in steps 2 and 3; and for Cd in steps 1,3, and 4. The worse RSDs for elements extracted in steps 1 and 2 can be explained by their lower concentrations in each step. The third step in the $\mathrm{BCR}$ protocol has cumbersome procedures compared to the other steps, which results in worse RSDs.

Figure 3 shows the recovery rates of the 38 elements. The recovery rate is the ratio of the total amount extracted in the four steps (sum of the four steps) and the bulk con- centration, which are summarized in Table 7 . The elements on the horizontal axis of Fig. 3 are arranged in ascending order of median value of the recovery rate determined for nine samples. The estimated recovery rate for samples ranged from $52 \%$ to $137 \%$ of the bulk composition (Fig. 3). Extreme overestimation of the recovery rate (137\%) occurred only for P in JSd-2. We suspected that the wavelength of $\mathrm{P}(213.618 \mathrm{~nm})$ was interfered by that of $\mathrm{Cu}(213.598 \mathrm{~nm})$; the $\mathrm{Cu}$ concentration in JSd-2 is very high. However, significant improvement did not occur even after correction of the interference. We thus pre- 
Table 4. (continued)

\begin{tabular}{|c|c|c|c|c|c|c|c|c|c|c|c|}
\hline \multirow[t]{2}{*}{ Elements } & \multirow[t]{2}{*}{ Unit } & \multicolumn{2}{|l|}{ JSd-3 } & \multicolumn{2}{|l|}{ JSd-4 } & \multicolumn{2}{|l|}{ JMs-1 } & \multicolumn{2}{|l|}{ JMs-2 } & \multicolumn{2}{|l|}{ JSO-1 } \\
\hline & & Mean $\pm 95 \% *$ & $N$ & Mean $\pm 95 \% *$ & $N$ & Mean $\pm 95 \% *$ & $N$ & Mean $\pm 95 \% *$ & $N$ & Mean $\pm 95 \% *$ & $N$ \\
\hline $\mathrm{Na}$ & $\mathrm{wt} \%$ & $0.0005 \pm 0.0004$ & 7 & $0.021 \pm 0.001$ & 7 & $0.049 \pm 0.003$ & 7 & $0.47 \pm 0.01$ & 6 & $0.0008 \pm 0.0005$ & 6 \\
\hline $\mathrm{Mg}$ & $\mathrm{wt} \%$ & $0.008 \pm 0.001$ & 7 & $0.63 \pm 0.01$ & 8 & $0.20 \pm 0.01$ & 8 & $0.22 \pm 0.01$ & 8 & $0.042 \pm 0.004$ & 9 \\
\hline $\mathrm{Al}$ & $\mathrm{wt} \%$ & $0.28 \pm 0.01$ & 8 & $1.03 \pm 0.04$ & 8 & $0.75 \pm 0.01$ & 7 & $0.75 \pm 0.02$ & 6 & $1.69 \pm 0.02$ & 7 \\
\hline$P$ & $\mathrm{mg} \mathrm{kg}^{-1}$ & $39 \pm 7$ & 6 & $386 \pm 38$ & 7 & $272 \pm 37$ & 6 & $1990 \pm 600$ & 7 & $52 \pm 14$ & 9 \\
\hline K & wt $\%$ & $0.014 \pm 0.001$ & 6 & $0.061 \pm 0.004$ & 8 & $0.112 \pm 0.001$ & 5 & $0.63 \pm 0.01$ & 6 & $0.006 \pm 0.001$ & 8 \\
\hline $\mathrm{Ca}$ & $\mathrm{wt} \%$ & $0.020 \pm 0.002$ & 8 & $0.225 \pm 0.008$ & 7 & $0.13 \pm 0.01$ & 8 & $0.95 \pm 0.02$ & 8 & $0.066 \pm 0.002$ & 8 \\
\hline $\mathrm{Sc}$ & $\mathrm{mg} \mathrm{kg}^{-1}$ & $<1.0$ & 3 & $1.3 \pm 0.3$ & 7 & $0.87 \pm 0.24$ & 6 & $1.1 \pm 0.2$ & 7 & $1.5 \pm 0.3$ & 8 \\
\hline V & $\mathrm{mg} \mathrm{kg}^{-1}$ & $4.2 \pm 2.2$ & 5 & $45 \pm 6$ & 7 & $25 \pm 4$ & 6 & $54 \pm 3$ & 5 & $46 \pm 3$ & 6 \\
\hline $\mathrm{Cr}$ & $\mathrm{mg} \mathrm{kg}^{-1}$ & $1.40 \pm 0.63$ & 4 & $356 \pm 31$ & 9 & $20.7 \pm 3.3$ & 7 & $1.85 \pm 0.80$ & 5 & $2.60 \pm 0.62$ & 6 \\
\hline $\mathrm{Mn}$ & $\mathrm{mg} \mathrm{kg}^{-1}$ & $268 \pm 12$ & 8 & $173 \pm 6$ & 9 & $135 \pm 7$ & 8 & $14780 \pm 50$ & 6 & $610 \pm 30$ & 9 \\
\hline $\mathrm{Fe}$ & $\mathrm{wt} \%$ & $0.22 \pm 0.03$ & 8 & $1.40 \pm 0.12$ & 9 & $0.91 \pm 0.06$ & 8 & $1.5 \pm 0.1$ & 7 & $0.61 \pm 0.06$ & 9 \\
\hline Co & $\mathrm{mg} \mathrm{kg}^{-1}$ & $4.1 \pm 0.2$ & 7 & $5.4 \pm 0.1$ & 8 & $3.8 \pm 0.3$ & 8 & $211 \pm 4$ & 7 & $11.4 \pm 0.5$ & 9 \\
\hline $\mathrm{Ni}$ & $\mathrm{mg} \mathrm{kg}^{-1}$ & $2.23 \pm 0.24$ & 7 & $42.7 \pm 1.6$ & 9 & $7.08 \pm 0.49$ & 8 & $202 \pm 3$ & 7 & $1.68 \pm 0.22$ & 8 \\
\hline $\mathrm{Cu}$ & $\mathrm{mg} \mathrm{kg}^{-1}$ & $92.5 \pm 11.8$ & 8 & $200 \pm 6$ & 7 & $23 \pm 3.2$ & 8 & $221 \pm 4$ & 6 & $2.87 \pm 0.80$ & 8 \\
\hline $\mathrm{Zn}$ & $\mathrm{mg} \mathrm{kg}^{-1}$ & $11.8 \pm 1.9$ & 8 & $781 \pm 44$ & 9 & $82.2 \pm 2.6$ & 7 & $51.7 \pm 2.4$ & 7 & $9.3 \pm 1.0$ & 8 \\
\hline $\mathrm{Rb}$ & $\mathrm{mg} \mathrm{kg}^{-1}$ & $6.4 \pm 0.5$ & 8 & $2.9 \pm 0.2$ & 9 & $4.6 \pm 0.3$ & 8 & $4.2 \pm 0.3$ & 6 & $0.90 \pm 0.07$ & 8 \\
\hline $\mathrm{Sr}$ & $\mathrm{mg} \mathrm{kg}^{-1}$ & $1.6 \pm 0.2$ & 7 & $33.4 \pm 0.7$ & 8 & $14.5 \pm 0.5$ & 6 & $122 \pm 4$ & 7 & $15.4 \pm 0.3$ & 8 \\
\hline Y & $\mathrm{mg} \mathrm{kg}^{-1}$ & $2.6 \pm 0.1$ & 7 & $6.2 \pm 0.2$ & 9 & $7.66 \pm 0.24$ & 8 & $206 \pm 3$ & 7 & $2.9 \pm 0.5$ & 9 \\
\hline Mo & $\mathrm{mg} \mathrm{kg}^{-1}$ & n.d. & - & $0.057 \pm 0.012$ & 8 & $0.041 \pm 0.010$ & 7 & $0.43 \pm 0.07$ & 8 & n.d. & 一 \\
\hline $\mathrm{Cd}$ & $\mathrm{mg} \mathrm{kg}^{-1}$ & $0.211 \pm 0.006$ & 6 & $1.90 \pm 0.25$ & 9 & $0.141 \pm 0.018$ & 7 & $0.307 \pm 0.007$ & 6 & $0.128 \pm 0.007$ & 8 \\
\hline $\mathrm{Cs}$ & $\mathrm{mg} \mathrm{kg}^{-1}$ & $1.8 \pm 0.1$ & 7 & $0.17 \pm 0.01$ & 9 & $0.14 \pm 0.01$ & 8 & $0.018 \pm 0.003$ & 6 & $0.23 \pm 0.01$ & 9 \\
\hline $\mathrm{Ba}$ & $\mathrm{mg} \mathrm{kg}^{-1}$ & $26.7 \pm 0.8$ & 8 & $379 \pm 12$ & 9 & $25.0 \pm 0.4$ & 8 & $415 \pm 5$ & 5 & $84 \pm 6$ & 9 \\
\hline $\mathrm{La}$ & $\mathrm{mg} \mathrm{kg}^{-1}$ & $2.1 \pm 0.1$ & 8 & $4.6 \pm 0.1$ & 9 & $6.0 \pm 0.2$ & 8 & $109 \pm 2$ & 7 & $1.4 \pm 0.2$ & 9 \\
\hline $\mathrm{Ce}$ & $\mathrm{mg} \mathrm{kg}^{-1}$ & $6.1 \pm 0.3$ & 7 & $8.4 \pm 0.3$ & 9 & $15 \pm 0.4$ & 8 & $107 \pm 5$ & 7 & $1.2 \pm 0.2$ & 9 \\
\hline $\operatorname{Pr}$ & $\mathrm{mg} \mathrm{kg}^{-1}$ & $0.60 \pm 0.04$ & 8 & $1.05 \pm 0.04$ & 9 & $1.75 \pm 0.04$ & 8 & $29.7 \pm 0.4$ & 7 & $0.23 \pm 0.04$ & 9 \\
\hline $\mathrm{Nd}$ & $\mathrm{mg} \mathrm{kg}^{-1}$ & $2.4 \pm 0.2$ & 8 & $4.3 \pm 0.2$ & 9 & $7.3 \pm 0.2$ & 8 & $126 \pm 3$ & 7 & $0.93 \pm 0.19$ & 9 \\
\hline Sm & $\mathrm{mg} \mathrm{kg}^{-1}$ & $0.57 \pm 0.04$ & 8 & $0.92 \pm 0.04$ & 9 & $1.69 \pm 0.05$ & 8 & $28.6 \pm 0.6$ & 7 & $0.19 \pm 0.04$ & 9 \\
\hline $\mathrm{Eu}$ & $\mathrm{mg} \mathrm{kg}^{-1}$ & $0.14 \pm 0.01$ & 8 & $0.24 \pm 0.02$ & 9 & $0.38 \pm 0.01$ & 8 & $6.6 \pm 0.2$ & 7 & $0.060 \pm 0.014$ & 9 \\
\hline Gd & $\mathrm{mg} \mathrm{kg}^{-1}$ & $0.56 \pm 0.02$ & 7 & $1.04 \pm 0.05$ & 9 & $1.76 \pm 0.04$ & 8 & $32.0 \pm 0.9$ & 7 & $0.26 \pm 0.06$ & 9 \\
\hline $\mathrm{Tb}$ & $\mathrm{mg} \mathrm{kg}^{-1}$ & $0.092 \pm 0.005$ & 8 & $0.16 \pm 0.01$ & 9 & $0.26 \pm 0.01$ & 8 & $4.9 \pm 0.1$ & 7 & $0.041 \pm 0.009$ & 9 \\
\hline Dy & $\mathrm{mg} \mathrm{kg}^{-1}$ & $0.57 \pm 0.04$ & 8 & $0.98 \pm 0.03$ & 9 & $1.55 \pm 0.02$ & 8 & $31.7 \pm 0.7$ & 7 & $0.29 \pm 0.06$ & 9 \\
\hline Но & $\mathrm{mg} \mathrm{kg}^{-1}$ & $0.11 \pm<0.01$ & 7 & $0.20 \pm 0.01$ & 9 & $0.29 \pm 0.01$ & 8 & $6.6 \pm 0.1$ & 7 & $0.066 \pm 0.014$ & 9 \\
\hline Er & $\mathrm{mg} \mathrm{kg}^{-1}$ & $0.29 \pm 0.02$ & 8 & $0.56 \pm 0.02$ & 9 & $0.76 \pm 0.02$ & 8 & $18.9 \pm 0.5$ & 7 & $0.20 \pm 0.04$ & 9 \\
\hline $\mathrm{Tm}$ & $\mathrm{mg} \mathrm{kg}^{-1}$ & $0.04 \pm 0.002$ & 7 & $0.075 \pm 0.004$ & 9 & $0.099 \pm 0.004$ & 8 & $2.58 \pm 0.06$ & 7 & $0.027 \pm 0.008$ & 9 \\
\hline $\mathrm{Yb}$ & $\mathrm{mg} \mathrm{kg}^{-1}$ & $0.26 \pm 0.01$ & 7 & $0.47 \pm 0.02$ & 9 & $0.61 \pm 0.02$ & 8 & $16.3 \pm 0.6$ & 7 & $0.17 \pm 0.04$ & 9 \\
\hline $\mathrm{Lu}$ & $\mathrm{mg} \mathrm{kg}^{-1}$ & $0.034 \pm 0.001$ & 7 & $0.066 \pm 0.003$ & 9 & $0.082 \pm 0.003$ & 8 & $2.51 \pm 0.09$ & 7 & $0.023 \pm 0.005$ & 8 \\
\hline $\mathrm{Pb}$ & $\mathrm{mg} \mathrm{kg}^{-1}$ & $27.5 \pm 1.4$ & 8 & $149 \pm 6$ & 9 & $37.1 \pm 0.8$ & 7 & $37.2 \pm 1.9$ & 8 & $2.17 \pm 0.33$ & 8 \\
\hline $\mathrm{Bi}$ & $\mathrm{mg} \mathrm{kg}^{-1}$ & $4.0 \pm 0.3$ & 8 & $0.071 \pm 0.009$ & 8 & $0.26 \pm 0.04$ & 8 & $0.010 \pm 0.002$ & 4 & n.d. & - \\
\hline
\end{tabular}

$N$, number of determinations; D.L., detection limit; n.d., not determinated; *95\% confidence interval.

sumed that the cause of the overestimation was analytical errors of concentration in the bulk composition and extractant in each step. Only 14 elements, such as Co and $\mathrm{Cu}$, had high enough recovery rates (median value $>90 \%$ ). Insignificant recovery rates were obtained for $\mathrm{Al}, \mathrm{Ca}, \mathrm{Sc}$, $\mathrm{Cr}, \mathrm{Rb}, \mathrm{Sr}, \mathrm{Y}, \mathrm{Mo}, \mathrm{Cs}$, Ln (La-Lu), and $\mathrm{Pb}$ (median values were $70-90 \%$ ). In this study, we used a $50 \mathrm{~mL}$ PFA tube to digest the step 3 residue without transporting it to a Teflon beaker. As a result, the contact area of residue with mixed acid in the PFA tube was much smaller than that in a Teflon beaker. Thus, these low recovery rates can be attributed in part to incomplete decomposition reaction in the PFA tube. Therefore, when we calculated the relative percentages of each extracted fraction for $\mathrm{Al}$, $\mathrm{Ca}, \mathrm{Sc}, \mathrm{Cr}, \mathrm{Rb}, \mathrm{Sr}, \mathrm{Y}, \mathrm{Mo}, \mathrm{Cs}, \mathrm{Ln}$, and $\mathrm{Pb}$, their concentrations in step 4 were calculated by the subtraction of the concentrations of steps 1,2 , and 3 from bulk concentrations. 
Table 5. The concentrations in step 3 obtained from BCR extraction procedure

\begin{tabular}{|c|c|c|c|c|c|c|c|c|c|c|}
\hline \multirow[t]{2}{*}{ Elements } & \multirow[t]{2}{*}{ Unit } & \multirow[t]{2}{*}{ D.L. } & \multicolumn{2}{|l|}{ BCR701 } & \multicolumn{2}{|l|}{ JLk-1 } & \multicolumn{2}{|l|}{ JSd-1 } & \multicolumn{2}{|l|}{ JSd-2 } \\
\hline & & & Mean $\pm 95 \% *$ & $N$ & Mean $\pm 95 \% *$ & $N$ & Mean $\pm 95 \% *$ & $N$ & Mean $\pm 95 \% *$ & $N$ \\
\hline $\mathrm{Na}$ & $\mathrm{wt} \%$ & 0.0001 & $0.004 \pm 0.001$ & 7 & $0.004 \pm 0.002$ & 6 & $0.010 \pm 0.003$ & 8 & $0.009 \pm 0.002$ & 8 \\
\hline $\mathrm{Mg}$ & $\mathrm{wt} \%$ & 0.00003 & $0.066 \pm 0.005$ & 6 & $0.047 \pm 0.003$ & 5 & $0.034 \pm 0.004$ & 6 & $0.037 \pm 0.004$ & 6 \\
\hline $\mathrm{Al}$ & $\mathrm{wt} \%$ & 0.001 & $0.14 \pm 0.03$ & 7 & $0.15 \pm 0.02$ & 6 & $0.07 \pm 0.01$ & 6 & $0.14 \pm 0.01$ & 6 \\
\hline$P$ & $\mathrm{mg} \mathrm{kg}^{-1}$ & 3 & $189 \pm 40$ & 7 & $100 \pm 60$ & 7 & $44 \pm 22$ & 7 & $109 \pm 59$ & 9 \\
\hline K & $\mathrm{wt} \%$ & 0.0001 & $0.014 \pm 0.003$ & 8 & $0.010 \pm 0.003$ & 6 & $0.012 \pm 0.005$ & 7 & $0.004 \pm 0.003$ & 5 \\
\hline $\mathrm{Ca}$ & $\mathrm{wt} \%$ & 0.0002 & $0.021 \pm 0.002$ & 8 & $0.031 \pm 0.002$ & 6 & $0.072 \pm 0.004$ & 7 & $0.036 \pm 0.004$ & 8 \\
\hline $\mathrm{Sc}$ & $\mathrm{mg} \mathrm{kg}^{-1}$ & 0.08 & $1.2 \pm 0.2$ & 7 & $2.8 \pm 0.4$ & 6 & $0.43 \pm 0.15$ & 6 & $1.8 \pm 0.3$ & 9 \\
\hline $\mathrm{Cr}$ & $\mathrm{mg} \mathrm{kg}^{-1}$ & 0.2 & $155 \pm 1$ & 5 & $3.57 \pm 0.49$ & 6 & $0.84 \pm 0.26$ & 7 & $2.31 \pm 0.22$ & 7 \\
\hline V & $\mathrm{mg} \mathrm{kg}^{-1}$ & 1 & $<3$ & 2 & $3.1 \pm 2.9$ & 4 & $4.4 \pm 2.8$ & 4 & $<4$ & 2 \\
\hline $\mathrm{Mn}$ & $\mathrm{mg} \mathrm{kg}^{-1}$ & 0.02 & $26.6 \pm 1.0$ & 5 & $102 \pm 11$ & 5 & $26.1 \pm 2.2$ & 5 & $26.9 \pm 7.2$ & 6 \\
\hline $\mathrm{Fe}$ & $\mathrm{wt} \%$ & 0.00005 & $0.17 \pm 0.08$ & 8 & $0.11 \pm 0.04$ & 6 & $0.016 \pm 0.009$ & 6 & $0.52 \pm 0.18$ & 7 \\
\hline Co & $\mathrm{mg} \mathrm{kg}^{-1}$ & 0.006 & $1.57 \pm 0.07$ & 7 & $2.5 \pm 0.14$ & 6 & $0.45 \pm 0.1$ & 7 & $5.7 \pm 0.7$ & 8 \\
\hline $\mathrm{Ni}$ & $\mathrm{mg} \mathrm{kg}^{-1}$ & 0.02 & $16.0 \pm 1.2$ & 8 & $3.93 \pm 0.35$ & 6 & $0.38 \pm 0.21$ & 7 & $15.6 \pm 2.1$ & 8 \\
\hline $\mathrm{Cu}$ & $\mathrm{mg} \mathrm{kg}^{-1}$ & 0.2 & $54.4 \pm 15$ & 8 & $13.1 \pm 3.5$ & 7 & $1.78 \pm 0.73$ & 5 & $549 \pm 75$ & 9 \\
\hline $\mathrm{Zn}$ & $\mathrm{mg} \mathrm{kg}^{-1}$ & 0.1 & $48.3 \pm 4.6$ & 8 & $16.1 \pm 2.3$ & 6 & $5.84 \pm 2.3$ & 6 & $132 \pm 12$ & 8 \\
\hline $\mathrm{Rb}$ & $\mathrm{mg} \mathrm{kg}^{-1}$ & 0.002 & $1.9 \pm 0.2$ & 7 & $2.0 \pm 0.2$ & 6 & $1.1 \pm 0.1$ & 6 & $0.50 \pm 0.18$ & 8 \\
\hline $\mathrm{Sr}$ & $\mathrm{mg} \mathrm{kg}^{-1}$ & 0.03 & $2.1 \pm 0.2$ & 8 & $1.4 \pm 0.2$ & 5 & $2.4 \pm 0.2$ & 7 & $3.8 \pm 0.5$ & 8 \\
\hline Y & $\mathrm{mg} \mathrm{kg}^{-1}$ & 0.003 & $1.4 \pm 0.2$ & 8 & $2.4 \pm 0.11$ & 7 & $1.8 \pm 0.4$ & 8 & $0.56 \pm 0.08$ & 8 \\
\hline Mo & $\mathrm{mg} \mathrm{kg}^{-1}$ & 0.01 & $0.88 \pm 0.28$ & 6 & $0.28 \pm 0.13$ & 5 & $0.23 \pm 0.02$ & 6 & $0.43 \pm 0.12$ & 8 \\
\hline $\mathrm{Cd}$ & $\mathrm{mg} \mathrm{kg}^{-1}$ & 0.003 & $0.312 \pm 0.049$ & 8 & $0.025 \pm 0.011$ & 6 & $0.016 \pm 0.006$ & 6 & $0.52 \pm 0.04$ & 8 \\
\hline Cs & $\mathrm{mg} \mathrm{kg}^{-1}$ & 0.003 & $0.29 \pm 0.07$ & 8 & $0.54 \pm 0.11$ & 6 & $0.049 \pm 0.026$ & 7 & $0.035 \pm 0.002$ & 6 \\
\hline $\mathrm{Ba}$ & $\mathrm{mg} \mathrm{kg}^{-1}$ & 0.1 & $14.3 \pm 0.4$ & 7 & $7.2 \pm 0.6$ & 6 & $7.1 \pm 0.8$ & 7 & $33 \pm 3$ & 8 \\
\hline $\mathrm{La}$ & $\mathrm{mg} \mathrm{kg}^{-1}$ & 0.004 & $1.2 \pm 0.1$ & 7 & $1.75 \pm 0.05$ & 6 & $0.50 \pm 0.19$ & 6 & $0.27 \pm 0.03$ & 7 \\
\hline $\mathrm{Ce}$ & $\mathrm{mg} \mathrm{kg}^{-1}$ & 0.006 & $3.6 \pm 0.3$ & 7 & $4.80 \pm 0.09$ & 6 & $0.77 \pm 0.36$ & 6 & $0.52 \pm 0.06$ & 7 \\
\hline $\operatorname{Pr}$ & $\mathrm{mg} \mathrm{kg}^{-1}$ & 0.0008 & $0.37 \pm 0.04$ & 7 & $0.54 \pm 0.02$ & 7 & $0.26 \pm 0.08$ & 7 & $0.09 \pm 0.02$ & 8 \\
\hline $\mathrm{Nd}$ & $\mathrm{mg} \mathrm{kg}^{-1}$ & 0.004 & $1.6 \pm 0.2$ & 7 & $2.25 \pm 0.07$ & 7 & $1.2 \pm 0.2$ & 7 & $0.33 \pm 0.03$ & 7 \\
\hline Sm & $\mathrm{mg} \mathrm{kg}^{-1}$ & 0.002 & $0.41 \pm 0.06$ & 7 & $0.60 \pm 0.02$ & 7 & $0.30 \pm 0.05$ & 7 & $0.089 \pm 0.009$ & 7 \\
\hline $\mathrm{Eu}$ & $\mathrm{mg} \mathrm{kg}^{-1}$ & 0.0006 & $0.064 \pm 0.011$ & 7 & $0.094 \pm 0.003$ & 7 & $0.083 \pm 0.014$ & 7 & $0.028 \pm 0.004$ & 8 \\
\hline Gd & $\mathrm{mg} \mathrm{kg}^{-1}$ & 0.002 & $0.39 \pm 0.08$ & 8 & $0.56 \pm 0.01$ & 7 & $0.33 \pm 0.06$ & 7 & $0.10 \pm 0.02$ & 8 \\
\hline $\mathrm{Tb}$ & $\mathrm{mg} \mathrm{kg}^{-1}$ & 0.0003 & $0.055 \pm 0.004$ & 6 & $0.089 \pm 0.002$ & 7 & $0.054 \pm 0.007$ & 6 & $0.017 \pm 0.003$ & 8 \\
\hline Dy & $\mathrm{mg} \mathrm{kg}^{-1}$ & 0.001 & $0.33 \pm 0.02$ & 6 & $0.53 \pm 0.02$ & 7 & $0.36 \pm 0.06$ & 7 & $0.12 \pm 0.02$ & 8 \\
\hline Но & $\mathrm{mg} \mathrm{kg}^{-1}$ & 0.0003 & $0.062 \pm 0.008$ & 7 & $0.102 \pm 0.004$ & 7 & $0.064 \pm 0.015$ & 8 & $0.022 \pm 0.002$ & 7 \\
\hline Er & $\mathrm{mg} \mathrm{kg}^{-1}$ & 0.0009 & $0.16 \pm 0.01$ & 6 & $0.27 \pm 0.01$ & 7 & $0.20 \pm 0.03$ & 7 & $0.061 \pm 0.004$ & 7 \\
\hline $\mathrm{Tm}$ & $\mathrm{mg} \mathrm{kg}^{-1}$ & 0.0002 & $0.023 \pm 0.004$ & 8 & $0.039 \pm 0.004$ & 7 & $0.029 \pm 0.004$ & 7 & $0.009 \pm 0.001$ & 7 \\
\hline $\mathrm{Yb}$ & $\mathrm{mg} \mathrm{kg}^{-1}$ & 0.001 & $0.17 \pm 0.01$ & 7 & $0.27 \pm 0.02$ & 7 & $0.20 \pm 0.03$ & 7 & $0.062 \pm 0.009$ & 8 \\
\hline $\mathrm{Lu}$ & $\mathrm{mg} \mathrm{kg}^{-1}$ & 0.0003 & $0.022 \pm 0.002$ & 7 & $0.036 \pm 0.003$ & 7 & $0.025 \pm 0.005$ & 8 & $0.008 \pm 0.002$ & 8 \\
\hline $\mathrm{Pb}$ & $\mathrm{mg} \mathrm{kg}^{-1}$ & 0.06 & $7.76 \pm 0.71$ & 7 & $2.52 \pm 0.63$ & 6 & $0.52 \pm 0.43$ & 5 & $3.57 \pm 0.74$ & 7 \\
\hline $\mathrm{Bi}$ & $\mathrm{mg} \mathrm{kg}^{-1}$ & 0.006 & $0.29 \pm 0.05$ & 7 & $0.066 \pm 0.007$ & 6 & $0.015 \pm 0.008$ & 7 & $0.11 \pm 0.017$ & 7 \\
\hline
\end{tabular}

Distribution of 38 elements in geochemical reference materials

Distribution of $\mathrm{Cr}, \mathrm{Ni}, \mathrm{Cu}, \mathrm{Zn}, \mathrm{Cd}$, and $\mathrm{Pb}$ in sediments Figure 4 shows the distributions of $\mathrm{Cr}, \mathrm{Ni}, \mathrm{Cu}, \mathrm{Zn}, \mathrm{Cd}$, and $\mathrm{Pb}$ concentrations in samples for the four fractions obtained from the BCR scheme. The BCR-701 sample was certified for the $\mathrm{Cr}, \mathrm{Ni}, \mathrm{Cu}, \mathrm{Zn}, \mathrm{Cd}$, and $\mathrm{Pb}$ concentrations for the three steps (Sutherland, 2010). The remaining metals amount in the residue could not be certified due to high variability between results obtained by different laboratories (Sahuquillo et al., 1999). Cr was extracted primarily from the residual fraction, from which mobility is low under environmental conditions (Morillo et al., 2004). However, the intensive feature was found in the distribution of $\mathrm{Cr}$ in BCR-701, JSd-4, and JMs-1. The high proportion of $\mathrm{Cr}$ in these samples was extracted in step $2(10-30 \%)$ and in step $3(20-50 \%)$. A similar distribution of $\mathrm{Cr}$ in urban soils was reported by Davidson et al. (1994, 2006). The Ni in JLk-1, JSd-1, and JSO-1 was dominantly associated with the residual fraction (step 4). However, a higher percentage of $\mathrm{Ni}$ was extracted in step $1(10-25 \%)$ and in step $2(35-70 \%)$ for JSd-2, JSd- 
Table 5. (continued)

\begin{tabular}{|c|c|c|c|c|c|c|c|c|c|c|c|}
\hline \multirow[t]{2}{*}{ Elements } & \multirow[t]{2}{*}{ Unit } & \multicolumn{2}{|l|}{ JSd-3 } & \multicolumn{2}{|l|}{ JSd-4 } & \multicolumn{2}{|l|}{ JMs-1 } & \multicolumn{2}{|l|}{ JMs-2 } & \multicolumn{2}{|l|}{ JSO-1 } \\
\hline & & Mean $\pm 95 \% *$ & $N$ & Mean $\pm 95 \% *$ & $N$ & Mean $\pm 95 \% *$ & $N$ & Mean $\pm 95 \% *$ & $N$ & Mean $\pm 95 \% *$ & $N$ \\
\hline $\mathrm{Na}$ & wt $\%$ & $0.002 \pm 0.001$ & 6 & $0.012 \pm 0.002$ & 8 & $0.020 \pm 0.003$ & 8 & $0.024 \pm 0.004$ & 8 & $0.002 \pm 0.001$ & 8 \\
\hline $\mathrm{Mg}$ & $\mathrm{wt} \%$ & $0.006 \pm 0.003$ & 6 & $0.14 \pm 0.04$ & 8 & $0.09 \pm 0.02$ & 7 & $0.104 \pm 0.005$ & 6 & $0.025 \pm 0.006$ & 8 \\
\hline $\mathrm{Al}$ & $\mathrm{wt} \%$ & $0.11 \pm 0.03$ & 7 & $0.51 \pm 0.11$ & 7 & $0.37 \pm 0.01$ & 6 & $0.63 \pm 0.06$ & 6 & $1.7 \pm 0.2$ & 8 \\
\hline $\mathrm{P}$ & $\mathrm{mg} \mathrm{kg}^{-1}$ & $10 \pm 7$ & 6 & $210 \pm 87$ & 7 & $73 \pm 28$ & 6 & $620 \pm 90$ & 6 & $165 \pm 33$ & 8 \\
\hline K & $\mathrm{wt} \%$ & $0.006 \pm 0.003$ & 7 & $0.011 \pm 0.004$ & 9 & $0.009 \pm 0.003$ & 6 & $0.18 \pm 0.01$ & 7 & $0.003 \pm 0.001$ & 8 \\
\hline $\mathrm{Ca}$ & $\mathrm{wt} \%$ & $0.008 \pm 0.002$ & 7 & $0.054 \pm 0.013$ & 8 & $0.056 \pm 0.001$ & 5 & $0.057 \pm 0.011$ & 7 & $0.013 \pm 0.001$ & 8 \\
\hline $\mathrm{Sc}$ & $\mathrm{mg} \mathrm{kg}^{-1}$ & $1.34 \pm 0.06$ & 5 & $3.1 \pm 0.7$ & 8 & $4.9 \pm 0.3$ & 8 & $1.3 \pm 0.1$ & 6 & $15 \pm 3$ & 9 \\
\hline $\mathrm{Cr}$ & $\mathrm{mg} \mathrm{kg}^{-1}$ & $1.53 \pm 0.54$ & 8 & $523 \pm 50$ & 9 & $32.2 \pm 2.8$ & 8 & $1.33 \pm 0.15$ & 6 & $14 \pm 2$ & 7 \\
\hline $\mathrm{V}$ & $\mathrm{mg} \mathrm{kg}^{-1}$ & $3.4 \pm 3.3$ & 3 & $6.9 \pm 1.5$ & 6 & $<3$ & 2 & $5.4 \pm 4.0$ & 4 & $68 \pm 21$ & 4 \\
\hline $\mathrm{Mn}$ & $\mathrm{mg} \mathrm{kg}^{-1}$ & $43 \pm 11$ & 7 & $45 \pm 14$ & 7 & $89 \pm 12$ & 7 & $363 \pm 40$ & 8 & $99 \pm 13$ & 8 \\
\hline $\mathrm{Fe}$ & wt $\%$ & $0.032 \pm 0.016$ & 6 & $0.16 \pm 0.05$ & 6 & $0.44 \pm 0.06$ & 6 & $0.022 \pm 0.013$ & 5 & $0.45 \pm 0.05$ & 8 \\
\hline Co & $\mathrm{mg} \mathrm{kg}^{-1}$ & $0.79 \pm 0.16$ & 7 & $2.0 \pm 0.4$ & 8 & $1.6 \pm 0.1$ & 7 & $4.0 \pm 0.7$ & 8 & $2.0 \pm 0.2$ & 7 \\
\hline $\mathrm{Ni}$ & $\mathrm{mg} \mathrm{kg}^{-1}$ & $3.31 \pm 0.87$ & 8 & $12.7 \pm 1.8$ & 8 & $5.58 \pm 0.69$ & 7 & $13.0 \pm 2.4$ & 8 & $1.62 \pm 0.29$ & 8 \\
\hline $\mathrm{Cu}$ & $\mathrm{mg} \mathrm{kg}^{-1}$ & $57.6 \pm 9.5$ & 7 & $152 \pm 19$ & 9 & $30.4 \pm 6.2$ & 8 & $8.6 \pm 2.5$ & 5 & $65 \pm 14$ & 9 \\
\hline $\mathrm{Zn}$ & $\mathrm{mg} \mathrm{kg}^{-1}$ & $11.8 \pm 4.8$ & 8 & $93 \pm 17$ & 9 & $23.6 \pm 2.2$ & 7 & $5.2 \pm 2.0$ & 5 & $4.98 \pm 0.81$ & 6 \\
\hline $\mathrm{Rb}$ & $\mathrm{mg} \mathrm{kg}^{-1}$ & $2.3 \pm 0.3$ & 7 & $1.4 \pm 0.1$ & 8 & $2.7 \pm 0.4$ & 8 & $6.4 \pm 0.6$ & 8 & $0.17 \pm 0.03$ & 9 \\
\hline $\mathrm{Sr}$ & $\mathrm{mg} \mathrm{kg}^{-1}$ & $0.80 \pm 0.18$ & 7 & $4.6 \pm 1.0$ & 8 & $3.4 \pm 0.7$ & 8 & $13 \pm 2$ & 8 & $2.5 \pm 0.5$ & 9 \\
\hline $\mathrm{Y}$ & $\mathrm{mg} \mathrm{kg}^{-1}$ & $0.45 \pm 0.12$ & 8 & $2.0 \pm 0.2$ & 9 & $1.6 \pm 0.1$ & 8 & $18 \pm 2$ & 8 & $13 \pm 1$ & 7 \\
\hline Mo & $\mathrm{mg} \mathrm{kg}^{-1}$ & $0.18 \pm 0.11$ & 8 & $0.28 \pm 0.12$ & 7 & $0.060 \pm 0.023$ & 6 & $0.95 \pm 0.58$ & 5 & $0.027 \pm 0.006$ & 5 \\
\hline $\mathrm{Cd}$ & $\mathrm{mg} \mathrm{kg}^{-1}$ & $0.12 \pm 0.03$ & 7 & $0.11 \pm 0.02$ & 8 & $0.012 \pm 0.008$ & 6 & $0.012 \pm 0.005$ & 5 & $0.029 \pm 0.012$ & 5 \\
\hline Cs & $\mathrm{mg} \mathrm{kg}^{-1}$ & $1.1 \pm 0.2$ & 7 & $0.28 \pm 0.05$ & 8 & $0.59 \pm 0.06$ & 8 & $0.21 \pm 0.02$ & 8 & $0.13 \pm 0.01$ & 9 \\
\hline $\mathrm{Ba}$ & $\mathrm{mg} \mathrm{kg}^{-1}$ & $5.6 \pm 0.8$ & 7 & $77 \pm 8$ & 8 & $0.31 \pm 0.29$ & 6 & $865 \pm 67$ & 6 & $11.9 \pm 1.2$ & 8 \\
\hline $\mathrm{La}$ & $\mathrm{mg} \mathrm{kg}^{-1}$ & $0.39 \pm 0.14$ & 8 & $1.45 \pm 0.06$ & 6 & $1.1 \pm 0.2$ & 7 & $10.4 \pm 0.8$ & 7 & $5.2 \pm 0.5$ & 7 \\
\hline $\mathrm{Ce}$ & $\mathrm{mg} \mathrm{kg}^{-1}$ & $1.8 \pm 0.5$ & 8 & $3.4 \pm 0.2$ & 6 & $3.5 \pm 0.3$ & 7 & $17 \pm 4$ & 8 & $5.3 \pm 1.5$ & 8 \\
\hline $\operatorname{Pr}$ & $\mathrm{mg} \mathrm{kg}^{-1}$ & $0.10 \pm 0.03$ & 7 & $0.54 \pm 0.08$ & 9 & $0.45 \pm 0.04$ & 7 & $3.1 \pm 0.5$ & 8 & $1.6 \pm 0.3$ & 9 \\
\hline $\mathrm{Nd}$ & $\mathrm{mg} \mathrm{kg}^{-1}$ & $0.44 \pm 0.14$ & 8 & $2.4 \pm 0.3$ & 9 & $2.0 \pm 0.2$ & 7 & $13 \pm 2$ & 8 & $8.3 \pm 0.5$ & 6 \\
\hline $\mathrm{Sm}$ & $\mathrm{mg} \mathrm{kg}^{-1}$ & $0.11 \pm 0.036$ & 8 & $0.64 \pm 0.08$ & 9 & $0.57 \pm 0.04$ & 7 & $3.4 \pm 0.5$ & 8 & $2.3 \pm 0.3$ & 7 \\
\hline $\mathrm{Eu}$ & $\mathrm{mg} \mathrm{kg}^{-1}$ & $0.023 \pm 0.008$ & 7 & $0.15 \pm 0.02$ & 9 & $0.13 \pm 0.01$ & 7 & $0.83 \pm 0.12$ & 8 & $0.58 \pm 0.11$ & 9 \\
\hline $\mathrm{Gd}$ & $\mathrm{mg} \mathrm{kg}^{-1}$ & $0.11 \pm 0.03$ & 8 & $0.58 \pm 0.07$ & 9 & $0.51 \pm 0.04$ & 7 & $3.4 \pm 0.5$ & 8 & $2.4 \pm 0.1$ & 6 \\
\hline $\mathrm{Tb}$ & $\mathrm{mg} \mathrm{kg}^{-1}$ & $0.015 \pm 0.004$ & 7 & $0.09 \pm 0.011$ & 9 & $0.074 \pm 0.007$ & 8 & $0.54 \pm 0.08$ & 8 & $0.39 \pm 0.05$ & 7 \\
\hline Dy & $\mathrm{mg} \mathrm{kg}^{-1}$ & $0.081 \pm 0.009$ & 6 & $0.54 \pm 0.07$ & 9 & $0.44 \pm 0.05$ & 8 & $3.5 \pm 0.5$ & 8 & $2.5 \pm 0.3$ & 7 \\
\hline Но & $\mathrm{mg} \mathrm{kg}^{-1}$ & $0.017 \pm 0.005$ & 7 & $0.10 \pm 0.01$ & 9 & $0.079 \pm 0.008$ & 8 & $0.68 \pm 0.09$ & 8 & $0.49 \pm 0.01$ & 5 \\
\hline $\mathrm{Er}$ & $\mathrm{mg} \mathrm{kg}^{-1}$ & $0.050 \pm 0.012$ & 7 & $0.28 \pm 0.03$ & 9 & $0.21 \pm 0.02$ & 8 & $2.0 \pm 0.3$ & 8 & $1.4 \pm 0.1$ & 6 \\
\hline $\mathrm{Tm}$ & $\mathrm{mg} \mathrm{kg}^{-1}$ & $0.0072 \pm 0.0025$ & 7 & $0.041 \pm 0.004$ & 9 & $0.030 \pm 0.003$ & 8 & $0.28 \pm 0.04$ & 8 & $0.20 \pm 0.02$ & 7 \\
\hline $\mathrm{Yb}$ & $\mathrm{mg} \mathrm{kg}^{-1}$ & $0.045 \pm 0.005$ & 6 & $0.27 \pm 0.03$ & 9 & $0.21 \pm 0.01$ & 7 & $1.9 \pm 0.3$ & 8 & $1.3 \pm 0.1$ & 6 \\
\hline $\mathrm{Lu}$ & $\mathrm{mg} \mathrm{kg}^{-1}$ & $0.0065 \pm 0.0022$ & 7 & $0.04 \pm 0.003$ & 9 & $0.029 \pm 0.002$ & 8 & $0.28 \pm 0.04$ & 8 & $0.18 \pm<0.01$ & 5 \\
\hline $\mathrm{Pb}$ & $\mathrm{mg} \mathrm{kg}^{-1}$ & $2.8 \pm 1.1$ & 7 & $46 \pm 11$ & 9 & $<0.5$ & 3 & $20.3 \pm 1.8$ & 6 & $4.18 \pm 0.85$ & 8 \\
\hline $\mathrm{Bi}$ & $\mathrm{mg} \mathrm{kg}^{-1}$ & $0.53 \pm 0.08$ & 7 & $0.44 \pm 0.08$ & 7 & $0.076 \pm 0.023$ & 7 & $0.035 \pm 0.008$ & 7 & $0.021 \pm 0.008$ & 8 \\
\hline
\end{tabular}

$N$, number of determinations; D.L., detection limit; $n . d .$, not determinated; $* 95 \%$ confidence interval.

4, and JMs-2. Figure 4 shows that $\mathrm{Cu}$ was extracted in every step, but especially large percentages of $\mathrm{Cu}$ for JSd2 and JSO-1 were extracted in step 3 . The presence of $\mathrm{Cu}$ in step 3 is consistent with the results of previous studies (Coetzee et al., 1995; Davidson et al., 1994; Morillo et al., 2004). These researchers suggested that $\mathrm{Cu}$ can readily form complexes with humic substances, which results in the high abundance in step 3. The distributions of the four fractions for $\mathrm{Zn}$ were largely different between the samples. Relatively high proportions of $\mathrm{Zn}$ were extracted in step $1(5-45 \%)$ and in step $2(10-65 \%)$. Cd is extremely unstable in sediments: it is easily dissolved into water and adsorbed on materials. Figure 4 shows that $\mathrm{Cd}$ was extracted principally in steps 1 and 2 for all samples. 15$80 \%$ of the total concentration of $\mathrm{Pb}$ was extracted in step $2 ; \mathrm{Pb}$ detected in step 1 was a minor species in sediments. These results are consistent with previous studies (Hudson-Edwards et al., 1996; Morillo et al., 2004), which suggested that $\mathrm{Fe}-\mathrm{Mn}$ hydrous oxides are important scavengers of $\mathrm{Pb}$ in sediments. The extraction per- 
Table 6. The concentrations in step 4 obtained from BCR extraction procedure

\begin{tabular}{|c|c|c|c|c|c|c|c|c|c|c|}
\hline \multirow[t]{2}{*}{ Elements } & \multirow[t]{2}{*}{ Unit } & \multirow[t]{2}{*}{ D.L. } & \multicolumn{2}{|l|}{ BCR701 } & \multicolumn{2}{|l|}{ JLk-1 } & \multicolumn{2}{|l|}{ JSd-1 } & \multicolumn{2}{|l|}{ JSd-2 } \\
\hline & & & Mean $\pm 95 \% *$ & $N$ & Mean $\pm 95 \% *$ & $N$ & Mean $\pm 95 \% *$ & $N$ & Mean $\pm 95 \% *$ & $N$ \\
\hline $\mathrm{Na}$ & wt $\%$ & 0.0001 & $1.48 \pm 0.12$ & 7 & $0.80 \pm 0.16$ & 7 & $2.11 \pm 0.27$ & 8 & $1.75 \pm 0.27$ & 9 \\
\hline $\mathrm{Mg}$ & $\mathrm{wt} \%$ & 0.00003 & $1.08 \pm 0.21$ & 8 & $0.77 \pm 0.15$ & 6 & $0.9 \pm 0.12$ & 8 & $1.39 \pm 0.22$ & 9 \\
\hline $\mathrm{Al}$ & $\mathrm{wt} \%$ & 0.0004 & $3.71 \pm 0.43$ & 6 & $4.9 \pm 1.0$ & 5 & $4.23 \pm 0.3$ & 5 & $3.40 \pm 0.46$ & 6 \\
\hline $\mathrm{P}$ & $\mathrm{mg} \mathrm{kg}^{-1}$ & 1 & $630 \pm 70$ & 6 & $440 \pm 116$ & 5 & $239 \pm 16$ & 5 & $281 \pm 46$ & 5 \\
\hline K & $\mathrm{wt} \%$ & 0.0003 & $1.97 \pm 0.06$ & 7 & $1.96 \pm 0.18$ & 6 & $1.62 \pm 0.05$ & 6 & $0.80 \pm 0.04$ & 6 \\
\hline $\mathrm{Ca}$ & $\mathrm{wt} \%$ & 0.0002 & $0.45 \pm 0.07$ & 6 & $0.13 \pm 0.04$ & 6 & $1.69 \pm 0.2$ & 6 & $0.98 \pm 0.15$ & 9 \\
\hline $\mathrm{Sc}$ & $\mathrm{mg} \mathrm{kg}^{-1}$ & 0.02 & $6.0 \pm 0.7$ & 6 & $10 \pm 3$ & 5 & $8.9 \pm 1.9$ & 6 & $12 \pm 1$ & 6 \\
\hline $\mathrm{Cr}$ & $\mathrm{mg} \mathrm{kg}^{-1}$ & 1 & $110 \pm 6$ & 7 & $46.6 \pm 6.5$ & 5 & $15.2 \pm 7.6$ & 6 & $74.4 \pm 3.3$ & 5 \\
\hline $\mathrm{V}$ & $\mathrm{mg} \mathrm{kg}^{-1}$ & 0.01 & $68 \pm 11$ & 7 & $92 \pm 4$ & 5 & $68 \pm 3$ & 6 & $114 \pm 6$ & 7 \\
\hline $\mathrm{Mn}$ & $\mathrm{mg} \mathrm{kg}^{-1}$ & 0.02 & $342 \pm 42$ & 6 & $365 \pm 59$ & 5 & $542 \pm 53$ & 6 & $545 \pm 46$ & 6 \\
\hline $\mathrm{Fe}$ & $\mathrm{wt} \%$ & 0.0001 & $2.70 \pm 0.08$ & 7 & $2.96 \pm 0.27$ & 6 & $2.75 \pm 0.10$ & 5 & $4.65 \pm 0.2$ & 7 \\
\hline Co & $\mathrm{mg} \mathrm{kg}^{-1}$ & 0.003 & $8.9 \pm 0.3$ & 7 & $9.5 \pm 0.6$ & 7 & $7.2 \pm 0.5$ & 8 & $13.2 \pm 0.4$ & 7 \\
\hline $\mathrm{Ni}$ & $\mathrm{mg} \mathrm{kg}^{-1}$ & 0.02 & $50.3 \pm 1.4$ & 7 & $26.9 \pm 1.0$ & 6 & $4.86 \pm 0.57$ & 7 & $30.3 \pm 2.4$ & 7 \\
\hline $\mathrm{Cu}$ & $\mathrm{mg} \mathrm{kg}^{-1}$ & 0.09 & $47.3 \pm 5.5$ & 6 & $31.8 \pm 1.9$ & 5 & $12.2 \pm 1.8$ & 7 & $112 \pm 8$ & 7 \\
\hline $\mathrm{Zn}$ & $\mathrm{mg} \mathrm{kg}^{-1}$ & 0.1 & $114 \pm 7$ & 7 & $90.7 \pm 8.3$ & 7 & $55.1 \pm 5.1$ & 7 & $148 \pm 28$ & 9 \\
\hline $\mathrm{Rb}$ & $\mathrm{mg} \mathrm{kg}^{-1}$ & 0.001 & $90 \pm 5$ & 6 & $69 \pm 18$ & 7 & $44 \pm 6$ & 6 & $16 \pm 1$ & 7 \\
\hline $\mathrm{Sr}$ & $\mathrm{mg} \mathrm{kg}^{-1}$ & 0.02 & $74 \pm 4$ & 7 & $35.3 \pm 7.5$ & 5 & $231 \pm 23$ & 5 & $123 \pm 14$ & 6 \\
\hline $\mathrm{Y}$ & $\mathrm{mg} \mathrm{kg}^{-1}$ & 0.003 & $6.6 \pm 1.2$ & 8 & $5.1 \pm 1.3$ & 6 & $5.3 \pm 0.5$ & 7 & $6.9 \pm 0.6$ & 8 \\
\hline Mo & $\mathrm{mg} \mathrm{kg}^{-1}$ & 0.01 & $3.21 \pm 0.40$ & 6 & $1.39 \pm 0.11$ & 5 & $0.14 \pm 0.06$ & 5 & $12.9 \pm 0.9$ & 7 \\
\hline $\mathrm{Cd}$ & $\mathrm{mg} \mathrm{kg}^{-1}$ & 0.002 & $0.210 \pm 0.019$ & 7 & $0.091 \pm 0.016$ & 6 & $0.031 \pm 0.007$ & 6 & $0.374 \pm 0.067$ & 7 \\
\hline Cs & $\mathrm{mg} \mathrm{kg}^{-1}$ & 0.0005 & $4.3 \pm 0.3$ & 7 & $9.2 \pm 0.7$ & 6 & $1.4 \pm 0.1$ & 8 & $0.65 \pm 0.06$ & 9 \\
\hline $\mathrm{Ba}$ & $\mathrm{mg} \mathrm{kg}^{-1}$ & 0.2 & $378 \pm 16$ & 7 & $344 \pm 56$ & 5 & $391 \pm 13$ & 7 & $350 \pm 30$ & 9 \\
\hline $\mathrm{La}$ & $\mathrm{mg} \mathrm{kg}^{-1}$ & 0.003 & $18.7 \pm 2.5$ & 7 & $5.3 \pm 2.2$ & 6 & $7.1 \pm 0.7$ & 6 & $5.2 \pm 0.7$ & 7 \\
\hline $\mathrm{Ce}$ & $\mathrm{mg} \mathrm{kg}^{-1}$ & 0.005 & $37.4 \pm 5.2$ & 7 & $12 \pm 4$ & 6 & $16 \pm 1$ & 6 & $9.7 \pm 1.6$ & 7 \\
\hline $\operatorname{Pr}$ & $\mathrm{mg} \mathrm{kg}^{-1}$ & 0.0004 & $4.7 \pm 0.6$ & 7 & $1.5 \pm 0.5$ & 6 & $1.8 \pm 0.1$ & 6 & $1.4 \pm 0.2$ & 7 \\
\hline $\mathrm{Nd}$ & $\mathrm{mg} \mathrm{kg}^{-1}$ & 0.002 & $18 \pm 2$ & 7 & $5.8 \pm 1.4$ & 6 & $7.1 \pm 0.5$ & 6 & $5.8 \pm 0.7$ & 7 \\
\hline $\mathrm{Sm}$ & $\mathrm{mg} \mathrm{kg}^{-1}$ & 0.0009 & $3.3 \pm 0.4$ & 7 & $1.2 \pm 0.3$ & 6 & $1.5 \pm 0.1$ & 7 & $1.4 \pm 0.1$ & 7 \\
\hline $\mathrm{Eu}$ & $\mathrm{mg} \mathrm{kg}^{-1}$ & 0.0004 & $0.49 \pm 0.05$ & 6 & $0.22 \pm 0.03$ & 6 & $0.45 \pm 0.05$ & 7 & $0.41 \pm 0.07$ & 7 \\
\hline $\mathrm{Gd}$ & $\mathrm{mg} \mathrm{kg}^{-1}$ & 0.001 & $2.6 \pm 0.3$ & 7 & $1.0 \pm 0.2$ & 6 & $1.3 \pm 0.1$ & 7 & $1.3 \pm 0.1$ & 7 \\
\hline $\mathrm{Tb}$ & $\mathrm{mg} \mathrm{kg}^{-1}$ & 0.0001 & $0.32 \pm 0.03$ & 7 & $0.16 \pm 0.02$ & 6 & $0.19 \pm 0.02$ & 7 & $0.21 \pm 0.03$ & 7 \\
\hline Dy & $\mathrm{mg} \mathrm{kg}^{-1}$ & 0.0007 & $1.7 \pm 0.2$ & 7 & $1.2 \pm 0.2$ & 6 & $1.2 \pm 0.1$ & 7 & $1.4 \pm 0.2$ & 7 \\
\hline Ho & $\mathrm{mg} \mathrm{kg}^{-1}$ & 0.0002 & $0.29 \pm 0.03$ & 7 & $0.26 \pm 0.03$ & 6 & $0.22 \pm 0.02$ & 7 & $0.28 \pm 0.03$ & 7 \\
\hline Er & $\mathrm{mg} \mathrm{kg}^{-1}$ & 0.0007 & $0.84 \pm 0.11$ & 6 & $0.87 \pm 0.1$ & 6 & $0.61 \pm 0.04$ & 7 & $0.84 \pm 0.1$ & 7 \\
\hline $\mathrm{Tm}$ & $\mathrm{mg} \mathrm{kg}^{-1}$ & 0.0002 & $0.10 \pm 0.01$ & 6 & $0.13 \pm 0.01$ & 6 & $0.081 \pm 0.013$ & 7 & $0.11 \pm 0.02$ & 7 \\
\hline $\mathrm{Yb}$ & $\mathrm{mg} \mathrm{kg}^{-1}$ & 0.0009 & $0.82 \pm 0.08$ & 6 & $1.0 \pm 0.1$ & 6 & $0.57 \pm 0.05$ & 7 & $0.78 \pm 0.10$ & 7 \\
\hline $\mathrm{Lu}$ & $\mathrm{mg} \mathrm{kg}^{-1}$ & 0.0001 & $0.10 \pm 0.01$ & 6 & $0.15 \pm 0.02$ & 6 & $0.072 \pm 0.012$ & 7 & $0.10 \pm 0.02$ & 7 \\
\hline $\mathrm{Pb}$ & $\mathrm{mg} \mathrm{kg}^{-1}$ & 0.07 & $21.7 \pm 2.2$ & 7 & $8.53 \pm 0.99$ & 7 & $6.9 \pm 1.0$ & 8 & $32.2 \pm 4$ & 8 \\
\hline $\mathrm{Bi}$ & $\mathrm{mg} \mathrm{kg}^{-1}$ & 0.004 & $0.91 \pm 0.24$ & 8 & $0.48 \pm 0.03$ & 6 & $0.068 \pm 0.034$ & 7 & $0.58 \pm 0.07$ & 8 \\
\hline
\end{tabular}

centage of $\mathrm{Pb}$ in step 3 was $5 \%$ or less, except for JSd-4, JMs-2, and JSO-1, which showed relatively high percentages $(18-24 \%)$.

Distribution of $S c, V, M n, F e, C o, M o$, and Bi in sediments Scandium and $\mathrm{V}$ were extracted primarily in the residual fraction (Fig. 5). Of steps 1-3, the relative percentages of Sc in step 3 and those of $\mathrm{V}$ in step 2 were the most dominant phases. As for $\mathrm{Mn}$, the geochemical reference materials had the most distinct proportion mutually. In JLk-1, Mn was found mainly in step 1 (around 60\%), which indicates that the mobility of Mn is high. For JSd1-4 and JMs-1, Mn was distributed equally between step $2(12-22 \%)$ and step $3(13-22 \%)$. In JMs-2, an extremely high proportion of Mn (90\%) was extracted in step 2 . The soil reference materials had similar proportions of $\mathrm{Mn}$ extracted in step $2(40-50 \%)$ and in step $4(35-45 \%)$. In contrast to Mn, only $10-30 \%$ of the total concentration of Fe was extracted in step 2. Iron was not extracted effectively in step 1 or step 3. Co had similar extraction results to $\mathrm{Mn}$, although a smaller percentage of Co for 
Table 6. (continued)

\begin{tabular}{|c|c|c|c|c|c|c|c|c|c|c|c|}
\hline \multirow[t]{2}{*}{ Elements } & \multirow[t]{2}{*}{ Unit } & \multicolumn{2}{|l|}{ JSd-3 } & \multicolumn{2}{|l|}{ JSd-4 } & \multicolumn{2}{|l|}{ JMs-1 } & \multicolumn{2}{|l|}{ JMs-2 } & \multicolumn{2}{|l|}{ JSO-1 } \\
\hline & & Mean $\pm 95 \% *$ & $N$ & Mean $\pm 95 \% *$ & $N$ & Mean $\pm 95 \% *$ & $N$ & Mean $\pm 95 \% *$ & $N$ & Mean $\pm 95 \% *$ & $\bar{N}$ \\
\hline $\mathrm{Na}$ & $\mathrm{wt} \%$ & $0.32 \pm 0.03$ & 8 & $0.96 \pm 0.13$ & 9 & $1.03 \pm 0.12$ & 8 & $0.73 \pm 0.09$ & 8 & $0.48 \pm 0.04$ & 8 \\
\hline $\mathrm{Mg}$ & $\mathrm{wt} \%$ & $0.67 \pm 0.09$ & 8 & $0.91 \pm 0.15$ & 9 & $1.00 \pm 0.14$ & 8 & $1.14 \pm 0.14$ & 8 & $1.13 \pm 0.13$ & 9 \\
\hline $\mathrm{Al}$ & $\mathrm{wt} \%$ & $3.56 \pm 0.24$ & 5 & $3.11 \pm 0.32$ & 6 & $4.10 \pm 0.43$ & 6 & $4.14 \pm 0.71$ & 6 & $3.34 \pm 0.27$ & 6 \\
\hline $\mathrm{P}$ & $\mathrm{mg} \mathrm{kg}^{-1}$ & $367 \pm 49$ & 5 & $1200 \pm 390$ & 8 & $427 \pm 97$ & 6 & $2880 \pm 180$ & 6 & $1790 \pm 320$ & 9 \\
\hline K & $\mathrm{wt} \%$ & $1.35 \pm 0.07$ & 8 & $0.82 \pm 0.06$ & 8 & $1.29 \pm 0.10$ & 8 & $1.02 \pm 0.04$ & 7 & $0.20 \pm 0.01$ & 8 \\
\hline $\mathrm{Ca}$ & $\mathrm{wt} \%$ & $0.22 \pm 0.03$ & 8 & $0.75 \pm 0.13$ & 7 & $0.41 \pm 0.01$ & 5 & $1.03 \pm 0.15$ & 8 & $1.08 \pm 0.11$ & 9 \\
\hline $\mathrm{Sc}$ & $\mathrm{mg} \mathrm{kg}^{-1}$ & $7.4 \pm 0.8$ & 8 & $9.2 \pm 1.2$ & 6 & $7.7 \pm 1.3$ & 6 & $19 \pm 2$ & 5 & $13 \pm 2$ & 7 \\
\hline $\mathrm{Cr}$ & $\mathrm{mg} \mathrm{kg}^{-1}$ & $24.2 \pm 2.2$ & 6 & $165 \pm 23$ & 6 & $57.8 \pm 4.0$ & 8 & $56.8 \pm 1.2$ & 6 & $46.7 \pm 2.6$ & 8 \\
\hline $\mathrm{V}$ & $\mathrm{mg} \mathrm{kg}^{-1}$ & $60 \pm 4$ & 7 & $85 \pm 6$ & 7 & $92 \pm 3$ & 6 & $111 \pm 4$ & 7 & $199 \pm 30$ & 8 \\
\hline $\mathrm{Mn}$ & $\mathrm{mg} \mathrm{kg}^{-1}$ & $697 \pm 69$ & 6 & $409 \pm 59$ & 6 & $351 \pm 21$ & 6 & $507 \pm 38$ & 6 & $640 \pm 70$ & 7 \\
\hline $\mathrm{Fe}$ & $\mathrm{wt} \%$ & $2.71 \pm 0.10$ & 7 & $3.63 \pm 0.16$ & 6 & $3.16 \pm 0.11$ & 6 & $5.57 \pm 0.34$ & 7 & $6.15 \pm 0.31$ & 7 \\
\hline Co & $\mathrm{mg} \mathrm{kg}^{-1}$ & $4.6 \pm 0.1$ & 7 & $8.3 \pm 0.6$ & 9 & $7.5 \pm 0.3$ & 7 & $11.1 \pm 0.7$ & 8 & $18.5 \pm 0.9$ & 8 \\
\hline $\mathrm{Ni}$ & $\mathrm{mg} \mathrm{kg}^{-1}$ & $12.4 \pm 0.6$ & 8 & $25.5 \pm 2.6$ & 8 & $35.4 \pm 0.8$ & 6 & $35.9 \pm 2.2$ & 7 & $36.1 \pm 1.1$ & 8 \\
\hline $\mathrm{Cu}$ & $\mathrm{mg} \mathrm{kg}^{-1}$ & $183 \pm 10$ & 7 & $51.0 \pm 7.3$ & 7 & $25.3 \pm 2.5$ & 7 & $209 \pm 6$ & 6 & $78.2 \pm 3.5$ & 8 \\
\hline $\mathrm{Zn}$ & $\mathrm{mg} \mathrm{kg}^{-1}$ & $103 \pm 4$ & 8 & $83 \pm 12$ & 9 & $62.7 \pm 8.5$ & 8 & $92.2 \pm 5.5$ & 7 & $86.2 \pm 6.3$ & 8 \\
\hline $\mathrm{Rb}$ & $\mathrm{mg} \mathrm{kg}^{-1}$ & $210 \pm 20$ & 6 & $33 \pm 3$ & 8 & $52 \pm 9$ & 5 & $36 \pm 5$ & 6 & $5.4 \pm 1.8$ & 7 \\
\hline $\mathrm{Sr}$ & $\mathrm{mg} \mathrm{kg}^{-1}$ & $39 \pm 5$ & 8 & $59 \pm 8$ & 7 & $53 \pm 2$ & 7 & $132 \pm 26$ & 8 & $62 \pm 6$ & 9 \\
\hline Y & $\mathrm{mg} \mathrm{kg}^{-1}$ & $5.3 \pm 1.1$ & 6 & $5.0 \pm 0.6$ & 7 & $5.1 \pm 1.2$ & 6 & $5.2 \pm 0.5$ & 7 & $3.8 \pm 0.4$ & 8 \\
\hline Mo & $\mathrm{mg} \mathrm{kg}^{-1}$ & $1.39 \pm 0.10$ & 8 & $4.00 \pm 0.33$ & 8 & $2.53 \pm 0.13$ & 8 & $18.8 \pm 2.4$ & 8 & $0.67 \pm 0.13$ & 7 \\
\hline $\mathrm{Cd}$ & $\mathrm{mg} \mathrm{kg}^{-1}$ & $0.183 \pm 0.032$ & 6 & $0.37 \pm 0.16$ & 8 & $0.065 \pm 0.009$ & 7 & $0.198 \pm 0.046$ & 7 & $0.067 \pm 0.010$ & 8 \\
\hline $\mathrm{Cs}$ & $\mathrm{mg} \mathrm{kg}^{-1}$ & $24 \pm 1$ & 6 & $2.5 \pm 0.2$ & 9 & $4.3 \pm 0.3$ & 8 & $2.3 \pm 0.2$ & 8 & $0.85 \pm 0.14$ & 9 \\
\hline $\mathrm{Ba}$ & $\mathrm{mg} \mathrm{kg}^{-1}$ & $365 \pm 29$ & 8 & $338 \pm 30$ & 9 & $233 \pm 22$ & 7 & $530 \pm 120$ & 6 & $72.6 \pm 8.7$ & 9 \\
\hline $\mathrm{La}$ & $\mathrm{mg} \mathrm{kg}^{-1}$ & $11 \pm 2$ & 7 & $5.5 \pm 0.7$ & 8 & $5.3 \pm 1.4$ & 7 & $7.9 \pm 1.4$ & 7 & $1.9 \pm 0.4$ & 9 \\
\hline $\mathrm{Ce}$ & $\mathrm{mg} \mathrm{kg}^{-1}$ & $22 \pm 4$ & 6 & $9.7 \pm 1.9$ & 8 & $11 \pm 3$ & 7 & $24 \pm 3$ & 6 & $7.0 \pm 1.2$ & 8 \\
\hline $\operatorname{Pr}$ & $\mathrm{mg} \mathrm{kg}^{-1}$ & $2.5 \pm 0.4$ & 8 & $1.3 \pm 0.2$ & 8 & $1.3 \pm 0.3$ & 7 & $1.9 \pm 0.2$ & 8 & $0.69 \pm 0.11$ & 9 \\
\hline $\mathrm{Nd}$ & $\mathrm{mg} \mathrm{kg}^{-1}$ & $9.4 \pm 1.4$ & 7 & $4.9 \pm 0.4$ & 7 & $5.0 \pm 0.9$ & 6 & $7.2 \pm 0.7$ & 7 & $3.1 \pm 0.5$ & 9 \\
\hline $\mathrm{Sm}$ & $\mathrm{mg} \mathrm{kg}^{-1}$ & $1.7 \pm 0.2$ & 8 & $0.99 \pm 0.08$ & 7 & $0.98 \pm 0.16$ & 6 & $1.5 \pm 0.1$ & 7 & $0.81 \pm 0.14$ & 9 \\
\hline $\mathrm{Eu}$ & $\mathrm{mg} \mathrm{kg}^{-1}$ & $0.35 \pm 0.04$ & 7 & $0.25 \pm 0.02$ & 9 & $0.23 \pm 0.03$ & 6 & $0.53 \pm 0.04$ & 8 & $0.25 \pm 0.02$ & 8 \\
\hline Gd & $\mathrm{mg} \mathrm{kg}^{-1}$ & $1.5 \pm 0.2$ & 7 & $0.91 \pm 0.08$ & 7 & $0.98 \pm 0.32$ & 6 & $1.4 \pm 0.1$ & 6 & $0.84 \pm 0.11$ & 8 \\
\hline $\mathrm{Tb}$ & $\mathrm{mg} \mathrm{kg}^{-1}$ & $0.19 \pm 0.02$ & 7 & $0.13 \pm 0.02$ & 8 & $0.13 \pm 0.03$ & 7 & $0.20 \pm 0.02$ & 7 & $0.14 \pm 0.01$ & 7 \\
\hline Dy & $\mathrm{mg} \mathrm{kg}^{-1}$ & $1.1 \pm 0.1$ & 8 & $0.89 \pm 0.1$ & 9 & $0.93 \pm 0.13$ & 7 & $1.3 \pm 0.1$ & 7 & $0.91 \pm 0.10$ & 8 \\
\hline Но & $\mathrm{mg} \mathrm{kg}^{-1}$ & $0.21 \pm 0.03$ & 8 & $0.18 \pm 0.02$ & 9 & $0.20 \pm 0.04$ & 7 & $0.24 \pm 0.03$ & 8 & $0.16 \pm 0.02$ & 9 \\
\hline Er & $\mathrm{mg} \mathrm{kg}^{-1}$ & $0.64 \pm 0.09$ & 8 & $0.58 \pm 0.06$ & 9 & $0.66 \pm 0.07$ & 7 & $0.73 \pm 0.08$ & 8 & $0.53 \pm 0.05$ & 8 \\
\hline $\mathrm{Tm}$ & $\mathrm{mg} \mathrm{kg}^{-1}$ & $0.091 \pm 0.014$ & 7 & $0.080 \pm 0.014$ & 7 & $0.10 \pm 0.02$ & 7 & $0.083 \pm 0.012$ & 7 & $0.070 \pm 0.005$ & 6 \\
\hline $\mathrm{Yb}$ & $\mathrm{mg} \mathrm{kg}^{-1}$ & $0.71 \pm 0.08$ & 8 & $0.63 \pm 0.06$ & 9 & $0.77 \pm 0.10$ & 7 & $0.69 \pm 0.06$ & 7 & $0.57 \pm 0.07$ & 8 \\
\hline $\mathrm{Lu}$ & $\mathrm{mg} \mathrm{kg}^{-1}$ & $0.10 \pm 0.02$ & 8 & $0.083 \pm 0.012$ & 9 & $0.11 \pm 0.02$ & 7 & $0.085 \pm 0.014$ & 7 & $0.077 \pm 0.009$ & 7 \\
\hline $\mathrm{Pb}$ & $\mathrm{mg} \mathrm{kg}^{-1}$ & $46.7 \pm 2.5$ & 8 & $40.0 \pm 7.3$ & 8 & $11.6 \pm 1.4$ & 8 & $23.8 \pm 3$ & 7 & $9.31 \pm 0.42$ & 6 \\
\hline $\mathrm{Bi}$ & $\mathrm{mg} \mathrm{kg}^{-1}$ & $7.43 \pm 0.51$ & 8 & $4.40 \pm 0.57$ & 7 & $0.62 \pm 0.06$ & 7 & $1.13 \pm 0.06$ & 8 & $0.17 \pm 0.03$ & 9 \\
\hline
\end{tabular}

$N$, number of determinations; D.L., detection limit; $n . d .$, not determinated; $* 95 \%$ confidence interval.

JLk-1 was extracted in step 1 and a larger percentage of Co for JSd-2-4 was extracted in step 2. Mo was barely extracted in steps 1,2 , and 3 ; it dominantly existed in the residual phase (Fig. 5). Slightly high proportions of Mo in step 3, especially for JSd-1, compared to those in steps 1 and 2 were found. Bi was extracted primarily in the residual fraction. JLk-1, JSd-1-3, and JMs- 1 had a significant amount of Bi extracted in step $2(25-55 \%)$.
Distribution of the alkali elements and the alkali earth elements Sodium, $\mathrm{K}, \mathrm{Rb}$, and $\mathrm{Cs}$ were extracted primarily in the residual fraction (Fig. 6). The extremely high proportion of Na detected in step 1 for JSd-4, JMs-1, and JMs-2 is explained by sea salt contamination because the sampling location of JSd-4 is near the sea and marine sediments were not desalinated. The alkali earth elements had different distributions from the alkali elements. 5- 

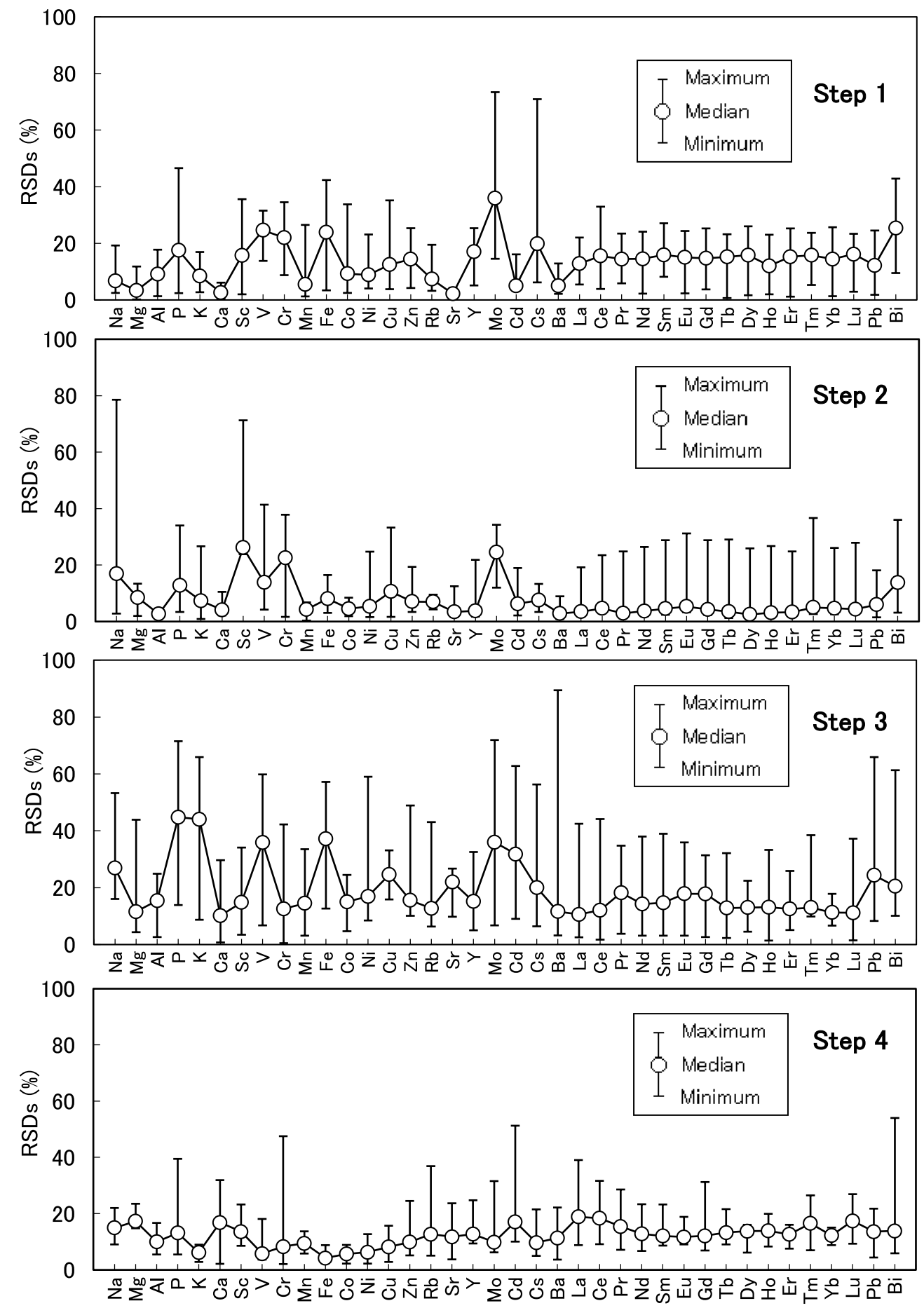

Fig. 2. The percentage relative standard deviation (RSD) used to exhibit reproducibility of repeated measurements of elemental concentrations in each step.

$70 \%$ of the total concentrations for $\mathrm{Ca}$ and $\mathrm{Sr}$ were found in step 1, which is explained by calcareous materials. A large amount of $\mathrm{Mg}$ was found in the residual phase (step 4), except for JSd-4, JMs-1, and JMs-2, which showed a high percentage in steps 1 and 2. The distribution of $\mathrm{Ba}$ among the four fractions varied between the samples. A significant proportion of Ba was extracted in both steps 1 and 2 for JSO-1; in step 2 for JLk-1, JSd-2, and JSd-4; and in both steps 2 and 3 for JMs-2. 


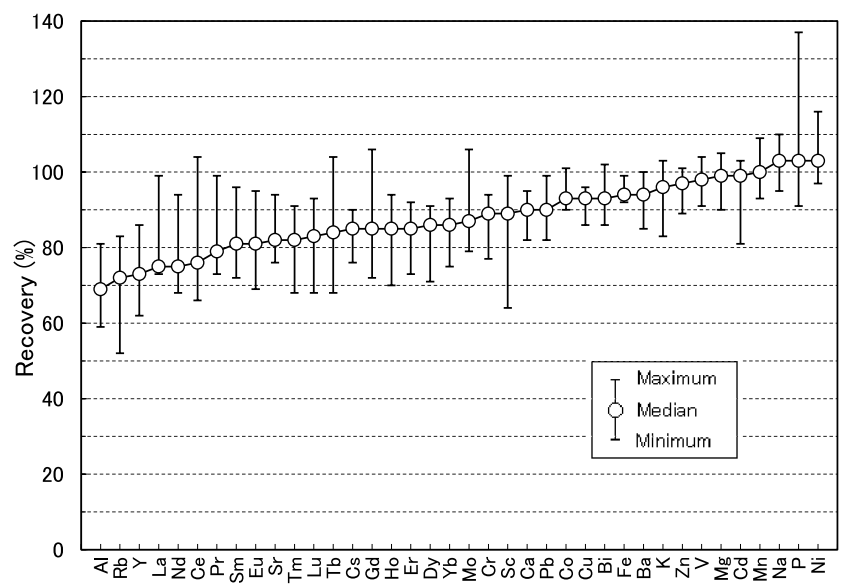

Fig. 3. Recovery rates of 38 elements for nine samples. The elements on the horizontal axis are arranged in ascending order of median value of recovery rate determined for nine samples.

Distribution of the other elements Most of the $\mathrm{Al}$ was present in the residual fraction (step 4), which accounts for nearly $90 \%$ of the total, except for JSO-1 (Fig. 7). JSO-1 had systematically higher proportions of $\mathrm{Al}$ in steps 2 and 3 than the other samples. In contrast, phosphorus in JSd-3 and JSO-1 was hardly extracted in steps 1-3. Except for these samples, extractable $\mathrm{P}$ was obtained principally in step $2(20-60 \%)$ and in step 3 (around 10\%). Figure 7 also shows that $\mathrm{Y}, \mathrm{La}, \mathrm{Ce}, \mathrm{Nd}, \mathrm{Eu}$, and $\mathrm{Yb}$ in the sediments were extracted largely in step $2(15-60 \%)$ among steps 1-3. However, JSO-1 had high proportions of $Y$ and $L n$ extracted in step $3(10-65 \%)$. The result of the soil reference material is not likely to be artifact from sequential extraction because similar results of La and $\mathrm{Ce}$ in stream sediments have been reported previously (Martin et al., 1998).

\section{Discussion}

In earlier sections, we discussed the determination of the value and enumerated the distribution characteristic of each element for geochemical reference materials. In this section, we elucidate how the speciation of elements extracted by the BCR protocol reflects their origin or sedimentary environment.

\section{Speciation of elements in lake sediments: JLk-1}

JLk-1 and CRM-701 are Japanese lake (Lake Biwa) and Italian lake (Lake Orta Piemonte) sediments, respectively. Although their sampling positions are distant, the distributions of elements, except for $\mathrm{Cr}, \mathrm{Mn}, \mathrm{Ni}, \mathrm{Cu}$, and $\mathrm{Zn}$, shown in Figs. 5-7 are quite similar mutually. JLk-1 was characterized by having a higher percentage of $\mathrm{Mn}$ and $\mathrm{Cd}$ extracted in step 1 and of $\mathrm{P}, \mathrm{Fe}, \mathrm{Y}, \mathrm{Ln}, \mathrm{Ba}, \mathrm{Pb}$, and $\mathrm{Bi}$ in step 2 than the other samples. These results correspond with the results of Nakashima (1982). He suggested that $\mathrm{Mn}$ and $\mathrm{Cd}$ were extracted mainly in the exchangeable fraction; that $\mathrm{Cu}, \mathrm{Zn}, \mathrm{As}$, and $\mathrm{Pb}$ were extracted largely in $\mathrm{Fe}-\mathrm{Mn}$ hydroxide fractions; and that $\mathrm{Ni}$ existed dominantly in the aluminosilicate fraction. Enrichments of $\mathrm{P}, \mathrm{Mn}, \mathrm{Ni}, \mathrm{Cu}, \mathrm{Zn}, \mathrm{As}, \mathrm{Cd}$, and $\mathrm{Pb}$ in surface sediments of Lake Biwa have been known for more than 30 years (e.g., Kobayashi et al., 1975). These were presumed to be caused by early diagenetic processes (Lynn and Bonatti, 1965; Robbins and Callender, 1975). Comprehensively, metals such as $\mathrm{Mn}, \mathrm{Fe}, \mathrm{Co}, \mathrm{Ni}, \mathrm{Cu}$, and $\mathrm{Zn}$ are dissolved at greater sediment depths under reducing conditions. They then diffuse upward and precipitate on the sediment surface under oxic conditions. In pelagic environments, Mn dioxide precipitates on surface sediments explain the high enrichment of Mn caused by diagenetic process (e.g., Chester, 1999). However, Ohta et al. (2006) reported that $95 \%$ of the $\mathrm{Mn}$ in JLk-1 exists as Mn(II). This result suggests that most of the Mn in JLk-1 was not present as Mn oxides but was weakly absorbed on mineral surfaces. Therefore, $\mathrm{P}, \mathrm{Fe}, \mathrm{Y}, \mathrm{Ln}, \mathrm{Ba}, \mathrm{Pb}$ and $\mathrm{Bi}$ seem to be strongly influenced by Fe hydroxides but not by Mn dioxide.

\section{Speciation of elements in stream sediments: JSd-1-4}

JSd-1 and JSd-3 are stream sediments collected from drainage basins covered by granitic rocks and sedimentary rocks, respectively. The lowest percentages of elements extracted at steps 1,2 , and 3 for these were obtained among geochemical reference materials, except for $\mathrm{P}, \mathrm{Co}, \mathrm{Cu}, \mathrm{Mo}$, and $\mathrm{Cd}$. These are typical cases of nonpolluted sediments. It is notable that a significant amount of Mo was extracted in step 3 for JSd-1 (about 50\%). Nevertheless, previous studies found that Mo in stream and marine sediments was barely extracted in steps 1,2 , and 3 and existed dominantly in the residual phase (Davidson et al., 1994; Yuan et al., 2004). A higher percentage of Mo in step 3 was also found in stream sediment samples that originated from granite rocks (Ohta et al., 2007). According to Ishihara (2002), the most common mineral that is composed mainly of Mo is molybdenite $\left(\mathrm{MoS}_{2}\right)$, and molybdenite occurs dominantly in granitic rocks. This report is consistent with the result of JSd-1 (granite origin) in this study.

JSd-2 is the stream sediment influenced by Hitachi $\mathrm{Cu}$ mines. Omori et al. (1986) reported that pyrite and chalcopyrite are major ores in the Hitachi mines. This is why a significant amount of $\mathrm{Cu}$ was extracted in step 3 for JSd-2 (about 50\%): $\mathrm{Cu}$ in the sample exists dominantly as copper sulfide. Indeed, the presence of copper sulfide in JSd-2 was confirmed using X-ray absorption fine structure spectroscopy, and $38 \%$ of the total $\mathrm{Cu}$ was found to 


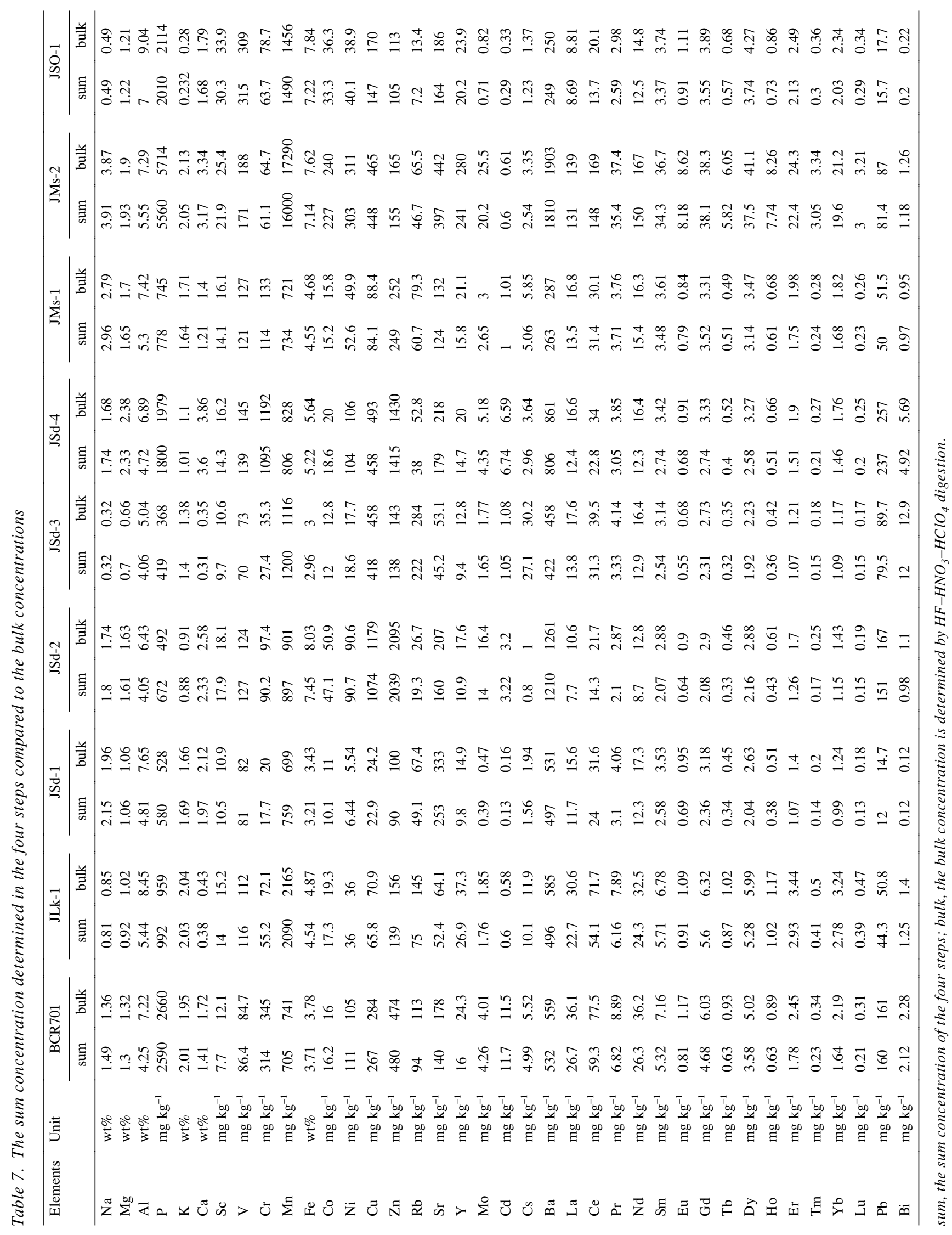



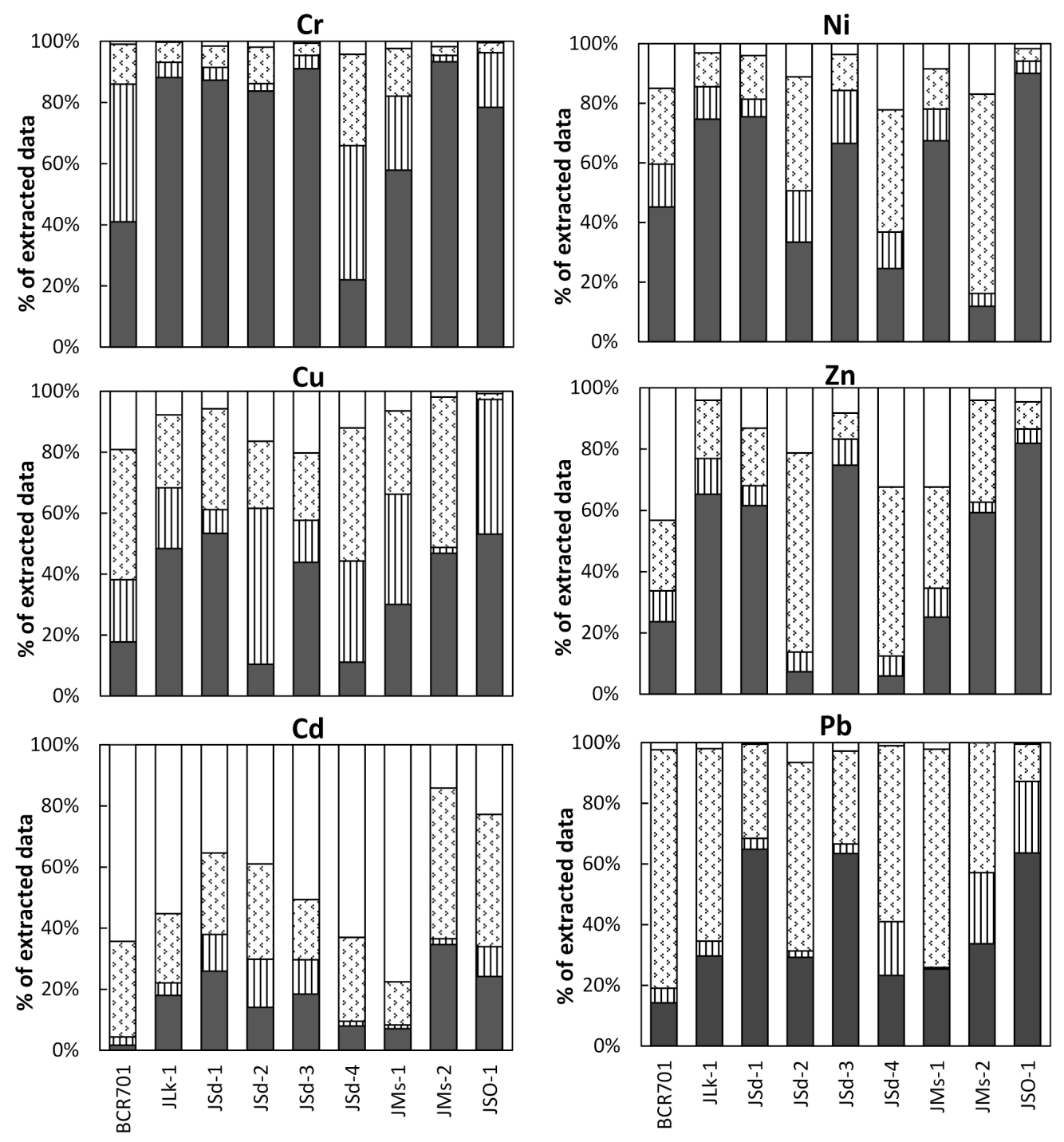

\section{$\square$ step $1 \quad$ : step 2 $\quad$ W step 3 $\quad \square$ step 4}

Fig. 4. Distribution of $\mathrm{Cr}, \mathrm{Ni}, \mathrm{Cu}, \mathrm{Zn}, \mathrm{Cd}$, and $\mathrm{Pb}$ concentrations in geochemical reference materials for four fractions obtained by the BCR scheme.

exist as a sulfide (Ohta et al., 2010). In contrast, Fe was scarcely extracted in step 3 (about 7\%). We assumed that the relative abundance ratio of pyrite and chalcopyrite to crystalline minerals containing Fe (e.g., hornblende, chlorite, epidote, and magnetite) in JSd-2 would be small. Actually, the GSJ Geochemical Reference Samples Database (https://gbank.gsj.jp/geostandards/) indicates that distinctive peaks of pyrite and chalcopyrite are not observed. As an example, considering the $\mathrm{Cu}$ in step 3 to be totally originated from chalcopyrite, the concentration of $\mathrm{Fe}$ from chalcopyrite is calculated to be only about 0.048 wt $\%$. This is considerably lower than the Fe concentra- tion of step 3 extractant (0.52 wt\%, Table 5). Another possible factor is that most of the pyrite was oxidized to Fe oxyhydroxides during the weathering process. In fact, a high percentage of $\mathrm{Fe}$ was extracted in step 2 (about $25 \%$ ). Aside from $\mathrm{Cu}$ and $\mathrm{Fe}$, JSd-2 had higher percentages of $\mathrm{Co}, \mathrm{Ca}$, and $\mathrm{Zn}$ in step 1 and of $\mathrm{Co}, \mathrm{Ni}, \mathrm{Zn}, \mathrm{Ba}$, and $\mathrm{Pb}$ in step 2 (Figs. 4, 5, and 6) compared to the other stream sediment reference materials not associated with mine activity (JSd-1 and JSd-3). These results suggest that $\mathrm{Ni}, \mathrm{Fe}, \mathrm{Cu}, \mathrm{Zn}, \mathrm{Co}$ and $\mathrm{Pb}$ released during the refining process adsorb onto mineral surfaces and bind to $\mathrm{Fe}$ oxides. The high $\mathrm{Ca}$ and $\mathrm{Ba}$ extraction in step 2 for JSd-2 

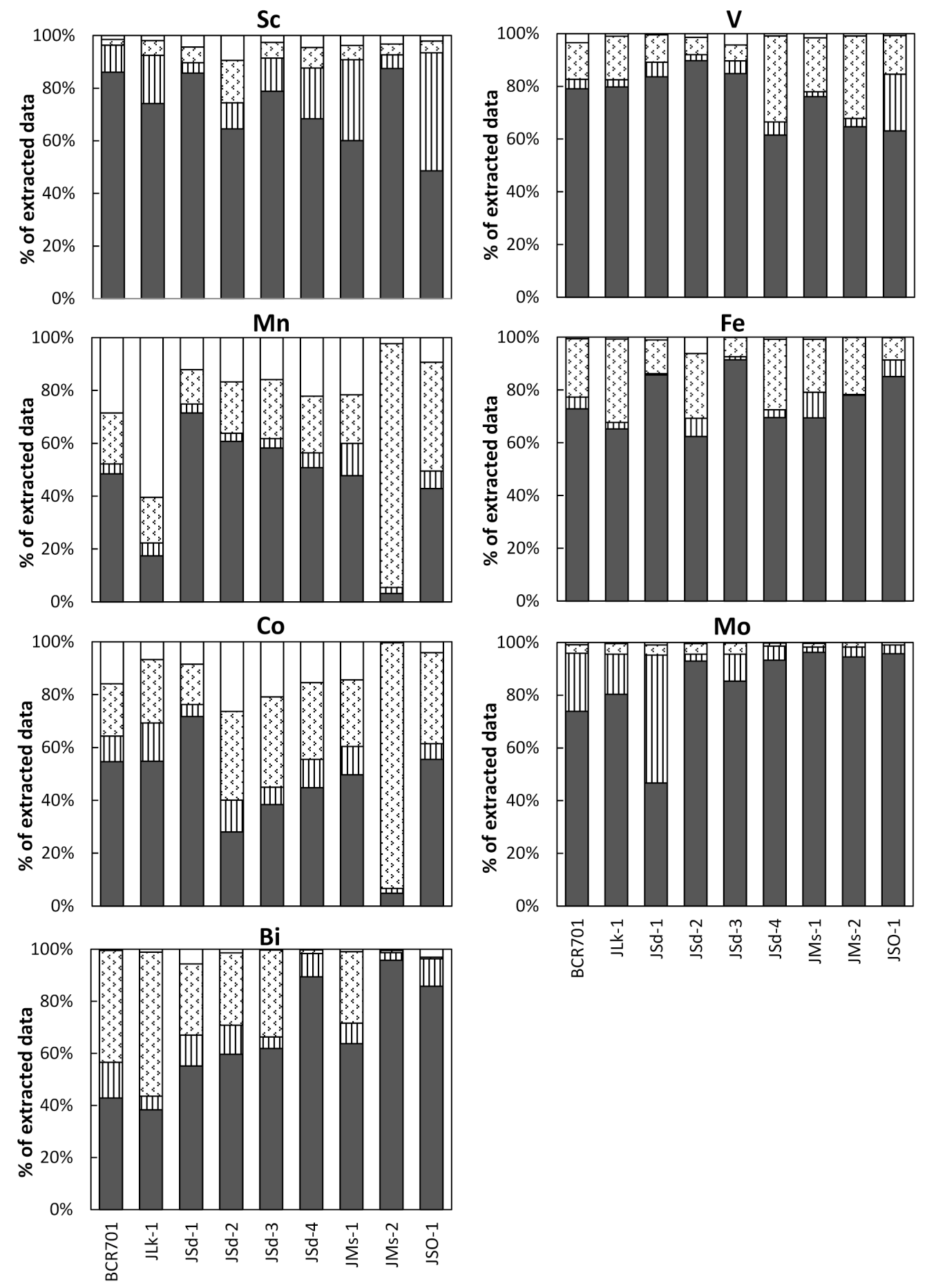

\section{$\begin{array}{llll}\square \text { step } 1 \quad \text { S step } 2 \quad \text { m step } 3 & \square \text { step } 4\end{array}$}

Fig. 5. Distribution of $\mathrm{Sc}, \mathrm{V}, \mathrm{Mn}, \mathrm{Fe}, \mathrm{Co}, \mathrm{Mo}$ and Bi concentrations in geochemical reference materials for four fractions obtained by the BCR scheme. 

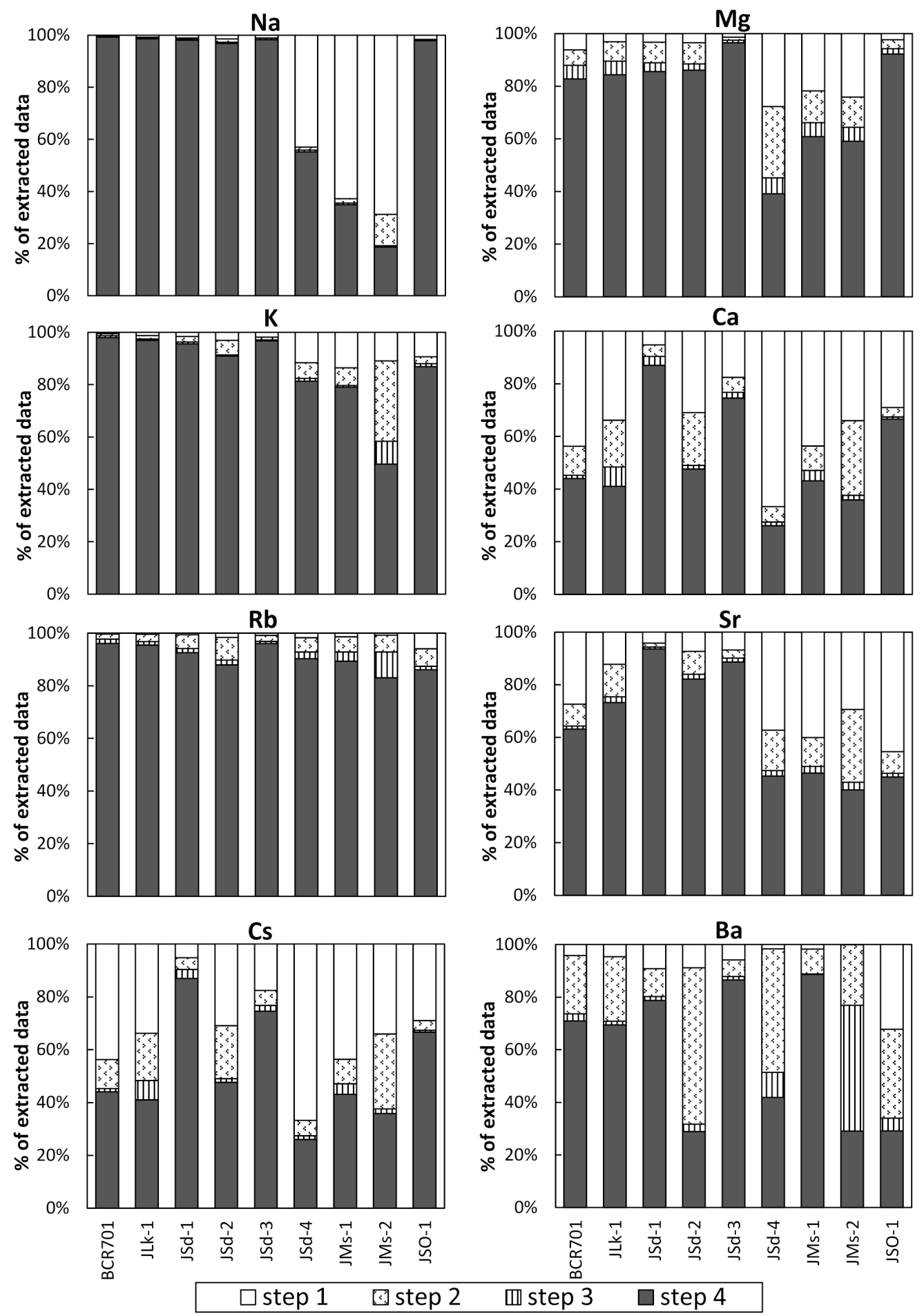

Fig. 6. Distribution of $\mathrm{Na}, \mathrm{Mg}, \mathrm{K}, \mathrm{Ca}, \mathrm{Rb}, \mathrm{Sr}, \mathrm{Cs}$ and $\mathrm{Ba}$ concentrations in geochemical reference materials for four fractions obtained by the BCR scheme.

is also explained by this same reason because the Hitachi mine also yields a large amount of skarn minerals and barite (Omori et al., 1986).

JSd-4 was collected from an urban area in the Kanto
Plain. Ohta et al. (2011) suggested that the stream sediments collected from urban areas in the Kanto Plain are significantly enriched in $\mathrm{P}, \mathrm{Cr}, \mathrm{Ni}, \mathrm{Cu}, \mathrm{Zn}, \mathrm{Sn}, \mathrm{Sb}$, $\mathrm{Hg}$, and $\mathrm{Pb}$ because of anthropogenic activities. Actually, 

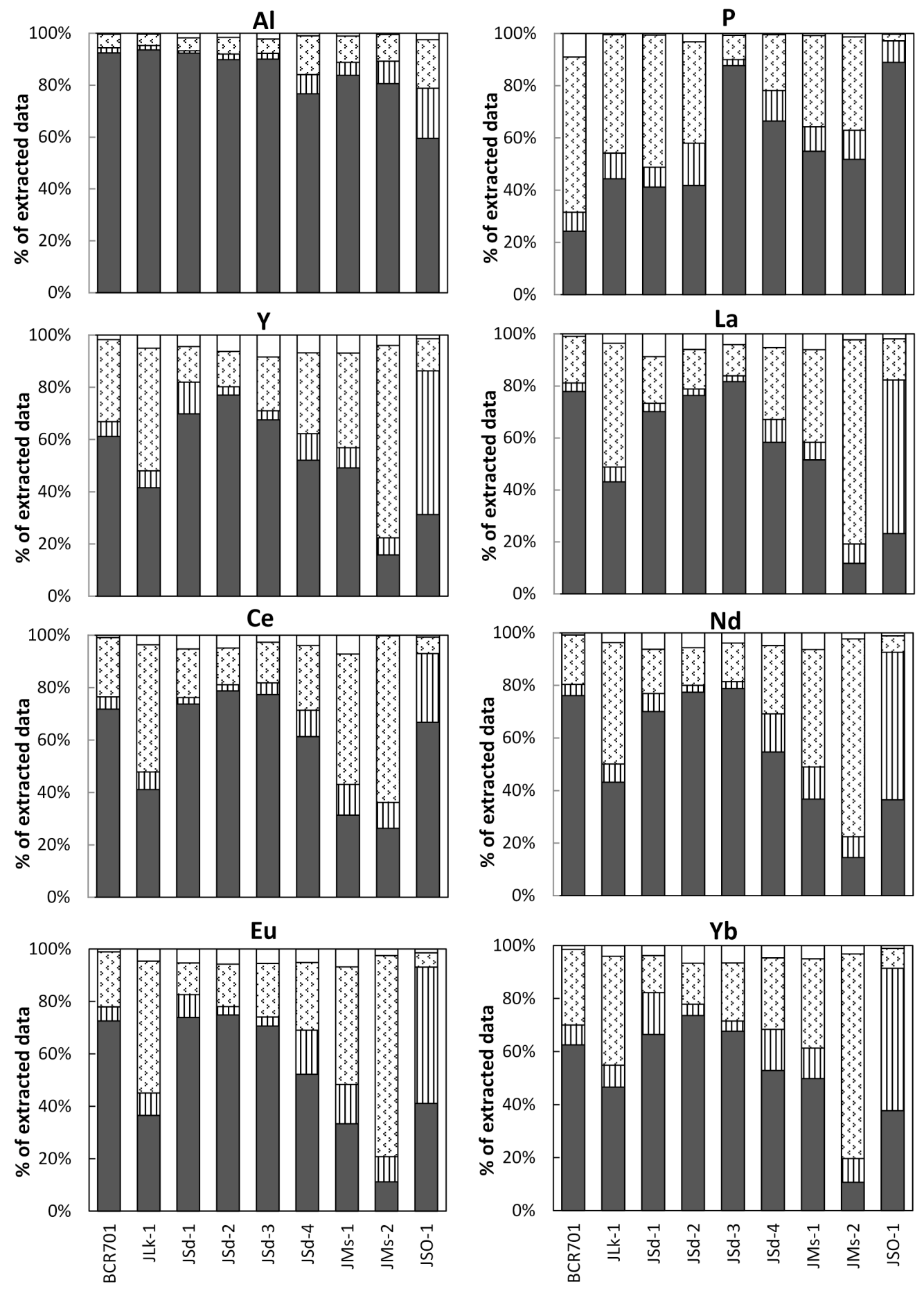

\section{$\begin{array}{llll}\square \text { step } 1 \quad \text { Step 2 } & \text { ⿴囗十 step 3 } & \square \text { step 4 }\end{array}$}

Fig. 7. Distribution of $\mathrm{Al}, \mathrm{P}, \mathrm{Y}, \mathrm{La}, \mathrm{Ce}, \mathrm{Nd}, \mathrm{Eu}$ and $\mathrm{Yb}$ concentrations in geochemical reference materials for four fractions obtained by the BCR scheme. 
JSd-4 has high concentrations of $\mathrm{P}\left(1,980 \mathrm{mg} \mathrm{kg}^{-1}\right), \mathrm{Cr}$ $\left(1,190 \mathrm{mg} \mathrm{kg}^{-1}\right)$, Ni (106 mg kg$\left.{ }^{-1}\right), \mathrm{Cu}\left(490 \mathrm{mg} \mathrm{kg}^{-1}\right), \mathrm{Zn}$ (1,430 $\left.\mathrm{mg} \mathrm{kg}^{-1}\right), \mathrm{Cd}\left(6.6 \mathrm{mg} \mathrm{kg}^{-1}\right)$, and $\mathrm{Pb}\left(260 \mathrm{mg} \mathrm{kg}^{-1}\right)$. Interestingly, the extraction results for these elements in JSd-4 were quite similar to those in JSd-2, except for $\mathrm{Cr}$. JSd-4 had higher fractions of $\mathrm{Ni}$ and $\mathrm{Zn}$ in step 1; of $\mathrm{V}$, $\mathrm{Cr}, \mathrm{Ni}, \mathrm{Cu}, \mathrm{Ba}, \mathrm{Zn}$, and $\mathrm{Pb}$ in step 2; and of $\mathrm{Cr}, \mathrm{Cu}$, and $\mathrm{Pb}$ in step 3. These results suggest that the $\mathrm{Ni}$ and $\mathrm{Zn}$ released by anthropogenic activities are weakly sorbed on mineral surfaces or bound to $\mathrm{Fe}-\mathrm{Mn}$ oxide phases; $\mathrm{Cr}$, $\mathrm{Cu}$, and $\mathrm{Pb}$ released by anthropogenic activities are bound both to Fe-Mn oxides and to organic materials. It is noteworthy that JSd-4 is also enriched in $\mathrm{Mg}, \mathrm{Ca}, \mathrm{V}$, and $\mathrm{Ba}$ compared with the other stream sediment materials in bulk composition (see Table 7). Significant amounts of $\mathrm{Mg}$, $\mathrm{Ca}$, and $\mathrm{Sr}$ in step 1 and of $\mathrm{Mg}, \mathrm{V}$, and $\mathrm{Ba}$ in step 2 are notable features. The enrichments and distribution features of these elements may be attributable to waste slag because the slag consists of oxides of $\mathrm{Mg}$ (3 5\%), $\mathrm{Al}$ (10 20\%), Si (30 55\%), and Ca (19 33\%) (Nagano et al., 2007; Nishi and Kawabata, 1990) and is associated with heavy metals such as $\mathrm{Cr}, \mathrm{Ni}, \mathrm{Cu}, \mathrm{Zn}$, and $\mathrm{Pb}$. The slag is reutilized for construction materials such as cement and asphalt pavement (Nagano et al., 2007).

\section{Speciation of elements in marine sediments: JMs-1 and $J M s-2$}

JMs-1 was collected from Tokyo Bay, which is surrounded by a highly populated urban area and large-scale industrial zone, and is thus affected by anthropogenic activities. It had speciation features similar to those of JSd-4 (stream sediment), whose sampling location is adjacent to Tokyo Bay. The distributions of Cr in JSd-4 and JMs-1 share a common thread of high extraction percentage in steps 2 and 3. A similar distribution of $\mathrm{Cr}$ in soil of areas affected by anthropogenic activities as in JSd-4 and JMs-1 was reported by Davidson et al. (1998). We assumed that the $\mathrm{Cr}$ was adsorbed on Fe-Mn oxides and humic substances, as discussed by Tokalioğlu and Kartal (2000). The oxidation state and chemical forms of $\mathrm{Cr}$ in sediments will be discussed in a following paper.

JMs-2 is pelagic sediment that was collected from the floor of the South Pacific Ocean. It is free from anthropogenic influences. JMs-2 had significant amounts of $\mathrm{P}$, $\mathrm{Fe}, \mathrm{Mn}, \mathrm{Ni}, \mathrm{Co}, \mathrm{Cu}, \mathrm{Zn}, \mathrm{Y}, \mathrm{Cd}, \mathrm{Ln}$, and $\mathrm{Pb}$ extracted at step 2, which is caused by diagenetic process, as described in Subsection "Speciation of elements in lake sediments: JLk-1". The high percentages of $\mathrm{Fe}$ and Mn extracted in step 2 were different from the case of JLk-1. The fact suggests that Fe hydroxides and Mn dioxides precipitate on surface sediments with coprecipitated or adsorbed $\mathrm{P}$, $\mathrm{Ni}, \mathrm{Co}, \mathrm{Cu}, \mathrm{Zn}, \mathrm{Y}, \mathrm{Cd}$, Ln, and Pb. Different from JMs-1, non-negligible amounts of $\mathrm{Na}, \mathrm{K}, \mathrm{Ca}, \mathrm{Sr}, \mathrm{Cs}$, and $\mathrm{Ba}$ were also extracted in step 2 for JMs-2. These elements were also incorporated into Mn dioxide. For instance, Moorby et al. (1984) reported that $\mathrm{Na}, \mathrm{Mg}, \mathrm{K}$, and Ca play an essential role in the crystallographic structure of birnessite, which is a Mn-dioxide mineral that is formed in many environments (of non-marine and marine hydrogenous, diagenetic, and hydrothermal origin). Similarly, $\mathrm{Ba}$ is also incorporated into Mn dioxides (Manceau et al., 2007). The high percentage of Ba extracted in step 3 may indicate that a portion of Ba strongly binds to organic materials in deep-sea sediments (Tsunogai et al., 1979).

\section{Speciation of elements in soil: JSO-1}

JSO-1 (forest soil) is highly enriched in organic matter (total carbon concentration is $8.91 \mathrm{wt} \%$ ) (Terashima et al., 2002). The higher proportion of $\mathrm{V}, \mathrm{Cr}, \mathrm{Cu}, \mathrm{Y}, \mathrm{Ln}$, and $\mathrm{Pb}$ in step 3 for JSO-1 suggests that these elements are present dominantly in organic phases. In fact, it has been confirmed that a significant proportion of the $\mathrm{V}$ and $\mathrm{Cu}$ bounded to humic acid can be extracted in step 3 (Coetzee et al., 1995; Davidson et al., 1994). Significant amounts of $\mathrm{Ca}, \mathrm{Sr}$, and $\mathrm{Ba}$ relative to the bulk concentrations were extracted in steps 1 and 2 . In contrast to marine sediments, calcareous materials are scarcely included in JSO-1 (Terashima et al., 2002). Accordingly, the extraction result suggests that a significant amount of $\mathrm{Ca}$, $\mathrm{Sr}$, and $\mathrm{Ba}$ are incorporated in clay minerals. Phosphate ions react with $\mathrm{Al}$ and $\mathrm{Fe}$ ions in volcanic ash soils to form insoluble compounds such as aluminum phosphate and iron phosphate. This is the reason that P in JSO-1 is scarcely extracted in steps 1,2 , and 3 . The soil reference material has higher Mn extraction in step $2(40 \%)$ than JSd-1-4. Ohta et al. (2006) suggested that $66 \%$ of the $\mathrm{Mn}$ in JSO-1 is in the trivalent state (Mn(III)) and that the rest is hexavalent (Mn(VI)). Almost all of the Mn(VI) and a part of the $\mathrm{Mn}(\mathrm{III})$ would be extracted in step 2 (relative extraction rate is $40 \%$ ).

\section{SUMMARY}

We determined the concentrations of 38 elements extracted by the BCR sequential extraction procedure for eight Japanese geochemical reference materials. We essentially followed the procedure proposed by BCR. Exceptionally, the mechanical shaker was operated at a higher speed $(80 \mathrm{rpm})$ than the proposed speed $(30 \mathrm{rpm})$ to keep samples suspended in experimental solutions during the extraction. The effect of the shaking speed and the precision and accuracy of the analytical data were confirmed using a certified reference material (BCR-701). As a result, the shaking speed does not affect the extraction percentage seriously but does slightly enhance the extraction of $\mathrm{Cr}, \mathrm{Mo}, \mathrm{Y}$, and lanthanide ( $\mathrm{Ln}$ ) in step 1. The estimated concentrations and repeatability errors of $\mathrm{Cr}, \mathrm{Ni}, \mathrm{Cu}, \mathrm{Zn}, \mathrm{Cd}$ and $\mathrm{Pb}$ extracted in respective steps 
were comparable to the certified values.

The RSDs obtained from repeated measurements for the 38 elements of eight geochemical reference materials were $<30 \%$ for steps 1 and 3 and $<20 \%$ for steps 2 and 4 . The RSDs obtained for low concentrations of elements extracted by the BCR scheme showed worse results. The third step in the BCR protocol is a cumbersome procedure that is different from the other steps, resulting in worse RSDs. The estimated recovery rates for samples ranged mostly from $70 \%$ to $100 \%$ of the bulk compositions. Insignificant recovery rates were obtained for $\mathrm{Al}$, $\mathrm{Sc}, \mathrm{Cr}, \mathrm{Rb}, \mathrm{Y}, \mathrm{Cs}$, and Ln. Unfortunately, the step 3 residue remaining in the PFA tubes was not decomposed completely using mixed acids.

The most dominant species of elements in the sediments were the residual phase (step 4). This result suggests that most elements are fundamentally stable in stream sediments. $\mathrm{Ca}$ and $\mathrm{Sr}$ in the geochemical reference materials were highly extracted in step 1 . Significantly high percentages of $\mathrm{V}, \mathrm{P}, \mathrm{Ni}, \mathrm{Y}, \mathrm{Ba}, \mathrm{Ln}$, and $\mathrm{Pb}$ were extracted in step 2. Mn, Co, Zn, and Cd had high proportions in steps 1 and 2; $\mathrm{Cu}$ was extracted significantly in all steps. Except for marine geochemical reference materials, the most dominant species of $\mathrm{Na}, \mathrm{K}, \mathrm{Rb}$, and $\mathrm{Cs}$ were the residual phase.

JLk-1 was characterized by having higher percentages of $\mathrm{Mn}$ and $\mathrm{Cd}$ extracted in step 1 because of diagenetic process. JSd-1 and JSd-3 had the lowest extraction of metals in steps $1-3$ among all samples. JSd-2, which is stream sediment influenced by $\mathrm{Cu}$ mines, had a significant proportion of $\mathrm{Cu}$ in step 3. It is suggested that JSd-4 was affected by anthropogenic activities, as evidenced by the larger percentages of $\mathrm{Cr}, \mathrm{Ni}, \mathrm{Cu}, \mathrm{Zn}$, and $\mathrm{Pb}$ extracted in steps 1-3 compared to the other samples. JMs-1 and JMs-2 had high proportions of $\mathrm{Na}, \mathrm{Mg}, \mathrm{K}, \mathrm{Ca}$, and $\mathrm{Sr}$ in step 1, which indicates the presence of sea salt and calcareous materials. In addition, significant amounts of $\mathrm{Mn}$, $\mathrm{Co}, \mathrm{Ni}, \mathrm{Cu}, \mathrm{Y}, \mathrm{Cd}$, and $\mathrm{Ln}$ were extracted in step 2 for JMs-2. This fact suggests that $\mathrm{Fe}-\mathrm{Mn}$ oxides are important carriers for these elements in pelagic sediments. $\mathrm{P}$ in JSO- 1 was scarcely extracted in steps 1,2 , and 3 because phosphate ions react with $\mathrm{Al}$ and $\mathrm{Fe}$ ions in volcanic ash soils to create insoluble compounds. The speciation of elements in reference materials correspond to their origin, mineral composition, and sedimentary environment. Therefore, the recommended values of 38 elements extracted by sequential extraction are beneficial for many geochemists who are conducting speciation geochemistry of sediments.

\section{REFERENCES}

Ando, A., Okai, T., Inouchi, Y., Igarashi, T., Sudo, S., Marumo, K., Itoh, S. and Terashima, S. (1990) JLk-1, JLs-1 and JDo-
1, GSJ rock reference samples of the "Sedimentary Rock Series": Bulletin of the Geological Survey of Japan. Bull. Geol. Surv. Japan 41, 27-48.

Chester, R. (1999) Marine Geochemistry. Blackwell Science Inc., U.K., 520 pp.

Coetzee, P. P., Gouws, K., Plüddemann, S., Yacoby, M., Howell, S. and den Drijver, L. (1995) Evaluation of sequential extraction procedures for metal speciation in model sediments. Water SA 21, 51-60.

Crosland, A. R., McGrath, S. P. and Lane, P. W. (1993) An interlaboratory comparison of a standardized EDTA extraction procedure for the analysis of available trace-elements in two quality-control soils. Int. J. Environ. Anal. Chem. 51, 153-160.

Davidson, C. M., Thomas, R. P., McVey, S. E., Perala, R., Littlejohn, D. and Ure, A. M. (1994) Evaluation of a sequential extraction procedure for the speciation of heavy metals in sediments. Anal. Chim. Acta 291, 277-286.

Davidson, C. M., Duncan, A. L., Littlejohn, D., Ure, A. M. and Garden, L. M. (1998) A critical evaluation of the three-stage BCR sequential extraction procedure to assess the potential mobility and toxicity of heavy metals in industriallycontaminated land. Anal. Chim. Acta 363, 45-55.

Davidson, C. M., Urquhart, G. J., Ajmone-Marsan, F., Biasioli, M., Duarte, A. D., Diaz-Barrientos, E., Grcman, H., Hossack, L., Hursthouse, A. S., Madrid, L., Rodrigues, S. and Zupan, M. (2006) Fractionation of potentially toxic elements in urban soils from five European cities by means of a harmonised sequential extraction procedure. Anal. Chim. Acta 565, 63-72.

Fauth, H., Hindel, R., Siewers, U. and Zinner, J. (1985) Geochemischer Atlas Bundesrepublik Deutschland. Bundesanstatt für Geowissenschaften and Rohstoffe (BGR), Hannover, 79 pp.

Gómez-Ariza, J. L., Giráldez, I., Sánchez-Rodas, D. and Morales, E. (1999) Metal readsorption and redistribution during the analytical fractionation of trace elements in oxic estuarine sediments. Anal. Chim. Acta 399, 295-307.

Howarth, R. J. and Thornton, I. (1983) Regional geochemical mapping and its application to environmental studies. Applied Environmental Geochemistry (Thornton, I., ed.), 4173, Academic Press London.

Hudson-Edwards, K. A., Macklin, M. G., Curtis, C. D. and Vaughan, D. J. (1996) Processes of formation and distribution of $\mathrm{Pb}-, \mathrm{Zn}-, \mathrm{Cd}-$, and $\mathrm{Cu}$-bearing minerals in the Tyne Basin, northeast England: Implications for metalcontaminated river systems. Environ. Sci. Technol. 30, 7280.

Imai, N., Terashima, S., Ohta, A., Mikoshiba, M., Okai, T., Tachibana, Y., Togashi, S., Matsuhisa, Y., Kanai, Y. and Kamioka, H. (2004) Geochemical Map of Japan. Geological Survey of Japan, AIST, 209 pp.

Imai, N., Terashima, S., Ohta, A., Mikoshiba, M., Okai, T., Tachibana, Y., Togashi, S., Matsuhisa, Y., Kanai, Y. and Kamioka, H. (2010) Elemental Distribution in JapanGeochemical Map of Japan- (Imai, N., ed.), Geological Survey of Japan, AIST, Tsukuba.

Ishihara, S. (2002) Chemical characteristics of the mineralized granitoids (1): Mo and W provinces of the Inner Zone of 
Southwest Japan. Bull. Geol. Surv. Japan 53(9/10), 657672.

Kobayashi, J., Muramoto, S., Nakashima, S., Teraoka, H. and Horie, S. (1975) Distribution of arsenic, cadmium, lead, zinc, copper, and manganese contained in the bottom sediment of Lake Biwa. Jpn. J. Limnol. 36, 6-15.

Lahermo, P., Ilmasti, M., Juntunen, R. and Taka, M. (1990) The Geochemical Atlas of Finland, Part 1: The Hydrogeochemical Mapping of Finnish Groundwater. Geological Survey of Finland, Espoopp.

Lis, J. and Pasieczna, A. (1995) Geochemical Atlas of Poland 1:2500000. Polish Geological Institute, Warszawapp.

López-Sánchez, J. F., Rubio, R. and Rauret, G. (1993) Comparison of two sequential extraction procedures for tracemetal partitioning in sediments. Int. J. Environ. Anal. Chem. 51, 113-121.

Lynn, D. C. and Bonatti, E. (1965) Mobility of manganese in diagenesis of deep-sea sediments. Mar. Geol. 3, 457-474.

Manceau, A., Lanson, M. and Geoffroy, N. (2007) Natural speciation of $\mathrm{Ni}, \mathrm{Zn}, \mathrm{Ba}$, and $\mathrm{As}$ in ferromanganese coatings on quartz using X-ray fluorescence, absorption, and diffraction. Geochim. Cosmochim. Acta 71, 95-128.

Marin, B., Valladon, M., Polve, M. and Monaco, A. (1997) Reproducibility testing of a sequential extraction scheme for the determination of trace metal speciation in a marine reference sediment by inductively coupled plasma-mass spectrometry. Anal. Chim. Acta 342, 91-112.

Martin, R., Sanchez, D. M. and Gutierrez, A. M. (1998) Sequential extraction of $\mathrm{U}, \mathrm{Th}, \mathrm{Ce}, \mathrm{La}$ and some heavy metals in sediments from Ortigas River, Spain. Talanta 46, 11151121.

Mester, Z., Cremisini, C., Ghiara, E. and Morabito, R. (1998) Comparison of two sequential extraction procedures for metal fractionation in sediment samples. Anal. Chim. Acta 359, 133-142.

Miller, J. N. and Miller, J. C. (2010) Statistics and Chemometrics for Analytical Chemistry. 6th ed., Pearson Education Ltd., England, $296 \mathrm{pp}$.

Moorby, S. A., Cronan, D. S. and Glasby, G. P. (1984) Geochemistry of hydrothermal Mn-oxide deposits from the S.W. Pacific island arc. Geochim. Cosmochim. Acta 48, 433441.

Morillo, J., Usero, J. and Gracia, I. (2004) Heavy metal distribution in marine sediments from the southwest coast of Spain. Chemosphere 55, 431-442.

Nagano, N., Takahashi, T., Tomita, K., Wakasugi, M., Kudo, K. and Omote, R. (2007) Study on chemical properties of molten slag derived from municipal solid waste. Reports of the Hokkaido Industrial Research Institute 306, 47-53.

Nakashima, S. (1982) Partitioning of heavy metals (Mn, Fe, $\mathrm{As}, \mathrm{Cd}, \mathrm{Pb}, \mathrm{Cu}, \mathrm{Zn}, \mathrm{Co}$ and $\mathrm{Ni}$ ) into selective chemical fractions in sediment cores from Lake Biwa. Jpn. J. Limnol. 43, 67-80.

Nishi, M. and Kawabata, K. (1990) Some basic and mechanical properties of iron and steel slags as base-course materials. J. Geol. Eng. 414, 89-98.

Ohta, A., Imai, N., Terashima, S. and Tachibana, Y. (2005) Application of multi-element statistical analysis for regional geochemical mapping in Central Japan. Appl. Geochem. 20,
1017-1037.

Ohta, A., Tsuno, H., Kagi, H., Kanai, Y., Nomura, M., Zhang, R., Terashima, S. and Imai, N. (2006) Chemical compositions and XANES speciations of Fe, $\mathrm{Mn}$ and $\mathrm{Zn}$ from aerosols collected in China and Japan during dust events. Geochem. J. 40, 363-376.

Ohta, A., Imai, N., Terashima, S. and Tachibana, Y. (2007) Preliminary study for speciation geochemical mapping using a sequential extraction method. Bull. Geol. Surv. Japan 58, 201-237.

Ohta, A., Kagi, H. and Ishibashi, H. (2010) Speciation study of copper in stream sediments. Photon Factroy Activitiy Report 27B, 39.

Ohta, A., Imai, N., Terashima, S. and Tachibana, Y. (2011) Regional geochemical mapping in eastern Japan including the nation's capital, Tokyo. Geochem.-Explor. Environ. Anal. 11, 211-223.

Omori, M., Hayama, Y. and Horiguchi, M. (1986) Regional Geology of Japan. Part 3 (KANTO). Kyoritsu Shuppan Co., $350 \mathrm{pp}$.

Quevauviller, P., Rauret, G., López-Sánchez, J. F., Rubio, R., Ure, A. and Muntau, H. (1997) Certification of trace metal extractable contents in a sediment reference material (CRM 601 ) following a three-step sequential extraction procedure. Sci. Total Environ. 205, 223-234.

Rauret, G., López-Sánchez, J. F., Sahuquillo, A., Rubio, R., Davidson, C., Ure, A. and Quevauviller, P. (1999) Improvement of the BCR three step sequential extraction procedure prior to the certification of new sediment and soil reference materials. J. Environ. Monit. 1, 57-61.

Rauret, G., López-Sánchez, J. F., Sahuquillo, A., Barahona, E., Lachica, M., Ure, A. M., Davidson, C. M., Gomez, A., Lück, D., Bacon, J., Yli-Halla, M., Muntau, H. and Quevauviller, P. (2000) Application of a modified BCR sequential extraction (three-step) procedure for the determination of extractable trace metal contents in a sewage sludge amended soil reference material (CRM 483), complemented by a threeyear stability study of acetic acid and EDTA extractable metal content. J. Environ. Monit. 2, 228-233.

Reimann, C., Äyräs, M., Chekushin, V., Bogatyrev, I., Boyd, R., Caritat, P. D., Dutter, R., Finne, T. E., Halleraker, J. H., Jæger, Ø., Kashulina, G., Lehto, O., Niskavaara, H., Pavlov, V., Räisänen, M. L., Strand, T. and Volden, T. (1998) Environmental Geochemical Atlas of the Central Barents Region. Geological Survey of Norway, Trondheim, Norway, $745 \mathrm{pp}$.

Robbins, J. A. and Callender, E. (1975) Diagenesis of manganese in Lake Michigan sediments. Am. J. Sci. 275, 512533.

Sahuquillo, A., López-Sánchez, J. F., Rubio, R., Rauret, G., Thomas, R. P., Davidson, C. M. and Ure, A. M. (1999) Use of a certified reference material for extractable trace metals to assess sources of uncertainty in the BCR three-stage sequential extraction procedure. Anal. Chim. Acta 382, 317327.

Salminen, R., Batista, M. J., Bidovec, M., Demetriades, A., De Viro, B., De Vos, W., Duris, M., Gilucis, A., Gregorauskiene, V., Halamic, J., Heitzmann, P., Lima, A., Jordan, G., Klaver, G., Klein, P., Lis, J., Locutura, J., Marsina, K., Mazreku, 
A., O’Connor, P. J., Olsson, S. Å., Ottesen, R.-T., Petersell, V., Plant, J. A., Reeder, S., Salpeteur, I., Sandström, H., Siewers, U., Steenfelt, A. and Tarvainen, T. (2005) Geochemical Atlas of Europe. Part 1-Background Information, Methodology and Maps. Geological Survey of Finland, Espoo, Finland, 526 pp.

Sutherland, R. A. (2010) BCR $^{\circledR}$-701: A review of 10-years of sequential extraction analyses. Anal. Chim. Acta 680, 1020.

Terashima, S., Ando, A., Okai, T., Kanai, Y., Taniguchi, M., Takizawa, F. and Itoh, S. (1990) Elemental concentrations in 9 new GSJ rock reference samples sedimentary-rock series. Geostand. Newsl. 14, 1-5.

Terashima, S., Imai, N., Taniguchi, M., Okai, T. and Nishimura, A. (2002) The preparation and preliminary characterisation of four new Geological Survey of Japan geochemical reference materials: Soils, JSO-1 and JSO-2; and marine sediments, JMS-1 and JMS-2. Geostand. Newsl. 26, 85-94.

Tessier, A., Campbell, P. G. C. and Bisson, M. (1979) Sequential extraction procedure for the speciation of particulate trace metals. Anal. Chem. 51, 844-851.

Thomas, R. P., Ure, A. M., Davidson, C. M., Littlejohn, D., Rauret, G., Rubio, R. and López-Sánchez, J. F. (1994) Threestage sequential extraction procedure for the determination of metals in river sediments. Anal. Chim. Acta 286, 423429.

Tokalioğlu, S. and Kartal, S. (2000) Determination of heavy metals and their speciation in lake sediments by flame atomic absorption spectrometry after a four-stage sequential extraction procedure. Anal. Chim. Acta 413, 33-40.

Tsunogai, S., Yonemaru, I. and Kusakabe, M. (1979) Post depositional migration of $\mathrm{Cu}, \mathrm{Zn}, \mathrm{Ni}, \mathrm{Co}, \mathrm{Pb}$ and $\mathrm{Ba}$ in deepsea sediments. Geochem. J. 13, 239-252.

Ure, A. M., Quevauviller, P., Muntau, H. and Griepink, B. (1993) Speciation of heavy metals in soils and sediments. An account of the improvement and harmonization of extraction techniques undertaken under the auspices of the BCR of the Commission of the European Communities. Int. J. Environ. Anal. Chem. 51, 135-151.

Usero, J., Gamero, M., Morillo, J. and Gracia, I. (1998) Comparative study of three sequential extraction procedures for metals in marine sediments. Environ. Int. 24, 487-496.

Weaver, T. A., Broxton, D. E., Bolivar, S. L. and Freeman, S. H. (1983) The Geochemical Atlas of Alaska: Compiled by the Geochemistry Group, Earth Sciences Division, Los Alamos National Laboratory. GJBX-32(83), Los Alamos, $57 \mathrm{pp}$.

Webb, J. S., Thornton, I., Thompson, M., Howarth, R. J. and Lowenstein, P. L. (1978) The Wolfson Geochemical Atlas of England and Wales. Clarendon Press, Oxford, 69 pp.

Xie, X. J., Mu, X. Z. and Ren, T. X. (1997) Geochemical mapping in China. J. Geochem. Explor. 60, 99-113.

Yuan, C. G., Shi, J. B., He, B., Liu, J. F., Liang, L. N. and Jiang, G. B. (2004) Speciation of heavy metals in marine sediments from the East China Sea by ICP-MS with sequential extraction. Environ. Int. 30, 769-783. 